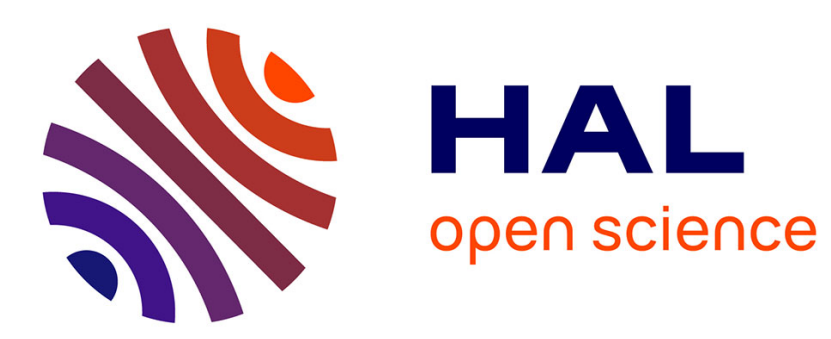

\title{
Anomalous rattling and single crystalline properties of the caged compound URu2Al10
}

\author{
R. Troć, M. Pasturel, M. Samsel-Czekala, R. Wawryk, Z. Gajek
}

\section{To cite this version:}

R. Troć, M. Pasturel, M. Samsel-Czekala, R. Wawryk, Z. Gajek. Anomalous rattling and single crystalline properties of the caged compound URu2Al10. Journal of Alloys and Compounds, 2018, 742, pp.656-669. 10.1016/j.jallcom.2018.01.319 . hal-01713499

HAL Id: hal-01713499

https://hal-univ-rennes1.archives-ouvertes.fr/hal-01713499

Submitted on 4 Jul 2018

HAL is a multi-disciplinary open access archive for the deposit and dissemination of scientific research documents, whether they are published or not. The documents may come from teaching and research institutions in France or abroad, or from public or private research centers.
L'archive ouverte pluridisciplinaire HAL, est destinée au dépôt et à la diffusion de documents scientifiques de niveau recherche, publiés ou non, émanant des établissements d'enseignement et de recherche français ou étrangers, des laboratoires publics ou privés. 


\title{
Anomalous rattling and single crystalline properties of the caged compound $\mathrm{URu}_{2} \mathrm{Al}_{10}$
}

\author{
R. Troć ${ }^{1 *}$, M. Pasturel ${ }^{2}$, M. Samsel-Czekała ${ }^{1}$, R. Wawryk ${ }^{1}$ and Z. Gajek ${ }^{1}$, \\ ${ }^{1}$ W. Trzebiatowski Institute of Low Temperature and Structure Research, Polish Academy of Sciences, Okólna 2, \\ 50-422 Wroctaw, Poland \\ ${ }^{2}$ Institut des Sciences Chimiques de Rennes, Chimie du Solide et Matériaux, Université Rennes 1, UMR CNRS \\ 6226, Campus de Beaulieu, 263 av. Général Leclerc, 35042 Rennes Cedex, France
}

* Corresponding author: Tel.: +48 713954 206;

E-mail address: R.Troc@int.pan.wroc.pl (R. Troć)

\begin{abstract}
We report on an experimental single-crystal study of $\mathrm{URu}_{2} \mathrm{Al}_{10}$, crystallizing in the $\mathrm{YbFe}_{2} \mathrm{Al}_{10}$ type orthorhombic structure, supplemented by the results of crystal field and band structure calculations. We investigated the magnetic, thermal and transport properties of this caged-type compound. Based on the local character of the $5 f^{2}$-electron configuration of the $\mathrm{U}^{4+}$ ion in $\mathrm{URu}_{2} \mathrm{Al}_{10}$, the effective crystal field $(\mathrm{CF})$ potential in the intermediate coupling form was estimated using the CF level scheme, composed only of singlets. This was carried out in a similar manner to that reported for $\mathrm{UFe}_{2} \mathrm{Al}_{10}$ [Phys. Rev. B 92 (2015) 104427]. The obtained scheme satisfactorily reproduces both the magnetic susceptibility (measured along the three main crystallographic directions) and the Schottky-type anomaly of the specific heat. The latter was estimated using the specific heat data of $\mathrm{ThRu}_{2} \mathrm{Al}_{10}$ as a phonon reference. In addition, the strong anisotropic behavior of the Seebeck coefficient measured along the three principal directions, and its low-temperature pronounced maxima, have been approximately explained by the $\mathrm{CF}$ effect. The latter dominates in the S-shaped temperature dependencies of the electrical resistivity, measured using the current flowing along the three main axes. However, the magnetoresistivity reveals an anisotropic electronic structure that could originate from a $c-f$ hybridization effect in an orthorhombic unit cell. This gives rise to the typical metallic character of $\mathrm{URu}_{2} \mathrm{Al}_{10}$, as is also the case for $\mathrm{UFe}_{2} \mathrm{Al}_{10}$. This behavior underlines the dual character of the $5 f$-electrons in these ternaries. In turn, the presence of low-frequency Einstein modes reflects the presence of regular rattling of the $\mathrm{U}^{4+}$ ion located in the $\left[\mathrm{Ru}_{4} \mathrm{Al}_{16}\right]$ cage. This rattling is, however, disturbed at low temperatures by applying an external magnetic field which causes strong scattering of the experimental electrical resistivity points. This effect is also anisotropic, as proved by a comparison of the resistivity results determined at zero and $9 \mathrm{~T}$ for a singlecrystalline sample of $\mathrm{URu}_{2} \mathrm{Al}_{10}$. The above effect also exists for isostructural $\mathrm{UFe}_{2} \mathrm{Al}_{10}$, but its anisotropy is less apparent.
\end{abstract}

Keywords:

Actinide alloys and compounds; Heat capacity; Magnetoresistance; Electron-phonon interactions; Kondo effect; Electronic band structure 


\section{Introduction}

Recently, the so-called cage-forming compounds such as skutterudites, fullerenes, clathrates, pyrochlores and others have attracted much attention, owing to their significant scientific and technological interest. All of these possess intriguing physical properties ranging from itinerant- to local-moment behavior. The latter usually leads to the crystal field effect and great magnetic anisotropy. They are also spin and valence fluctuators, exhibit heavy fermion- and non-Fermi liquid states, showing either conventional or unconventional superconductivity. Some of them are magnetically or multipolarly ordered. An origin of the large variety of behaviors in cage-like compounds is the electron-phonon coupling between Einstein-like phonons and conduction electrons [1-3].

A large group of rare earth $(R)$ or actinide $(A n)$ based ternary intermetallic compounds has also been categorized as cage-based systems; these have a general chemical formula $A_{\mathrm{x}} T_{\mathrm{y}} M_{\mathrm{z}}$, where the $\mathrm{x}: \mathrm{y}: \mathrm{z}$ stoichiometry is either 1:2:10 [4] or 1:2:20 [5], contain a transition element ( $T$ ), and where $M$ is either $\mathrm{Al}$ or $\mathrm{Al}, \mathrm{Zn}$ and $\mathrm{Cd}$, respectively.

This category arises when a so-called guest atom $(A=R$ or $A n)$ with either a metallic or ionic character is only weakly bound to the surrounding atoms, which form a polyhedron of $(M, T)$ or only $(M)$ atoms around the central $A$ atom. The most characteristic feature of such compounds is an oversized rigid cage formed by ligands with closed outer electronic shells in relation to the small size of the central atom. As a result the $A$ atom can move around inside the cage. In consequence, this situation leads to the so-called "rattling" effect, i.e. the anharmonic motion of the central atom. This effect can in turn be responsible for e.g. (i) high thermoelectric performance, and (ii) emerging superconductivity (SC) [6,7], or at least an enhancement of the superconducting transition temperature, as recently shown, for instance, in the $R \mathrm{~V}_{2} \mathrm{Al}_{20}$ aluminides ( $R=\mathrm{Sc}, \mathrm{Lu}$ or $\mathrm{Y}$ ) [8]. Here, one should also mention certain metallic binaries such as $\mathrm{ZrB}_{12}$ and $\mathrm{LuB}_{12}$, which crystallize into a cubic $\mathrm{UB}_{12}$ cage type of structure, or their solid solutions $\left(\mathrm{Zr}_{1-\mathrm{x}} \mathrm{Lu}_{\mathrm{x}}\right) \mathrm{B}_{12}$, and which are also superconductors $[9,10]$. Note that uranium dodecaboride is not a superconductor, even down to $0.4 \mathrm{~K}$ [11 and references therein].

The $A_{\mathrm{x}} T_{\mathrm{y}} M_{\mathrm{z}}$ ternaries mentioned above adopt orthorhombic $\mathrm{YbFe}_{2} \mathrm{Al}_{10}$ and cubic $\mathrm{CeCr}_{2} \mathrm{Al}_{20}$ types of structure, and form an atomic polyhedron around the guest atom $A$ that contains either $16 M+$ $4 T$ or $16 M$ atoms, respectively [4,5]. The main feature of these two families of ternaries is the large $A-$ $A$ nearest-neighbor distances, where $d_{\mathrm{A}-\mathrm{A}} \sim 5$ to $6 \AA$. In skutterudites, such distances are even larger, reaching about $7 \AA$. The large separations between guest atoms in these caged compounds significantly reduce the overlap between the $5 f$ electron wave functions, e.g. in the case of uraniumbearing compounds, which allows us to observe the crystal-field (CF) splitting of levels quite distinctly. Due to the small ratio of the $A$ atoms in the "molecule" containing 13 atoms, these dilute $5 f$ electron systems display no magnetic ordering, down to the lowest measured temperatures. This fact opens the way for possible detailed studies on the other type of existing inter- or intra-atomic interactions, performed at the lowest temperatures using advanced techniques.

In terms of actinides, $A n T_{2} \mathrm{Al}_{20}$ aluminides based on $\mathrm{Th}$ and $\mathrm{U}$ have been found for $\mathrm{Ti}, \mathrm{Nb}, \mathrm{Ta}$, $\mathrm{W}, \mathrm{V}, \mathrm{Cr}$ and $\mathrm{Mn}[5,12-16]$. All of these show metallic properties; only $\mathrm{UMn}_{2} \mathrm{Al}_{20}$ exhibits an itinerant ferromagnetic order [13,14] carried by $\mathrm{Mn}$ atoms, while all the remaining ternaries are Pauli paramagnets down to the lowest measured temperatures. Additionally, the $A n_{\mathrm{x}} \mathrm{V}_{2} \mathrm{Al}_{20}(A n=\mathrm{Th}, \mathrm{U}, \mathrm{Np}$,

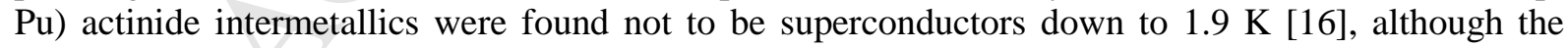
rattling of the $A n$-atoms inside their $\mathrm{Al}$ atom cage is large (their Einstein temperatures are around 20 $\mathrm{K})$. Similar magnetic properties to the above aluminides are also exhibited by some $\mathrm{U} T_{2} \mathrm{Zn}_{20}$ ternaries containing $T=\mathrm{Fe}$ or $\mathrm{Ru}$ [17]. On the other hand, the phases $\mathrm{UCo}_{2} \mathrm{Zn}_{20}$ and $\mathrm{URh}_{2} \mathrm{Zn}_{20}$ were found to behave as paramagnetic heavy-fermion systems with well-localized $5 f$-electrons [18]. No long-range magnetic ordering (even down to $350 \mathrm{mK}$ ) was also found for $\mathrm{UIr}_{2} \mathrm{Zn}_{20}$ [19]. The localized character of these systems and the lack of any magnetic order is the consequence of the large $\mathrm{U}-\mathrm{U}$ distance of about $6 \AA$ and hence very weak $f$-ligand hybridization.

Of the former series of ternaries, the $\mathrm{Ce}_{2} \mathrm{Al}_{10}(T=\mathrm{Fe}, \mathrm{Ru}, \mathrm{Os})$ systems have been most extensively studied in recent years. These aluminides exhibit Kondo semiconducting behavior [20]. Moreover, although the shortest distance between Ce atoms is as large as $5.2 \AA$, those ternaries with $\mathrm{Ru}$ and Os are characterized by an unconventional antiferromagnetic (AFM) ordering from the point 
of view of their high values of transition temperatures (around about $30 \mathrm{~K}$ ) [20, 21 and references therein]. It appears that although the separation between Ce ions is large, the anisotropic hybridization effect between conduction and $4 f$ electrons ( $c-f)$ (being the highest along the $a$ axis) is likely to be responsible for the formation of such an unusual long-range magnetic order. This effect is also the origin of the unusually large anisotropy in the temperature dependencies of the susceptibilities and transport properties of the orthorhombic system; this is also indicated by a large CF effect in the presence of strong anisotropic $c-f$ hybridization [22]. Regardless of the fact that in the paramagnetic region $\chi_{\mathrm{a}}>\chi_{\mathrm{c}}>\chi_{\mathrm{b}}$, the AFM ordered moment of $0.4(0.3) \mu_{\mathrm{B}}$ in $T=\mathrm{Ru}(\mathrm{Os})$ is, however, parallel to the $c$-axis. The smallness of this moment was explained by Strigari et al. [23,24] as being due solely to the CF effect, which is much higher than that found in the common Ce-intermetallics. Thus, the strongest $c-f$ hybridization along the $a$-axis prevents the alignment of the magnetic moment along this axis. In addition, the optical conductivity indicates the presence of anisotropic hybridization gaps opening for the $a$-, $b$ - and $c$-axes [25,26]. On the other hand, $\mathrm{CeFe}_{2} \mathrm{Al}_{10}$ has been classified as an intermediate system, and shows the highest degree of $c-f$ hybridization of these three systems [26]. Nevertheless, its Sommerfeld coefficient $\gamma(0 \mathrm{~K})=14 \mathrm{~mJ} \mathrm{~mol}^{-1} \mathrm{~K}^{-2}$ is lower than that of $\operatorname{LaFe}_{2} \mathrm{Al}_{10}(18$ $\mathrm{mJ} \mathrm{mol}^{-1} \mathrm{~K}^{-2}$ ); this is probably enhanced by the spin fluctuation effect (see below).

Furthermore, another ternary aluminide member of this series, $\mathrm{YFe}_{2} \mathrm{Al}_{10}$, has recently been intensively investigated due to its proximity to ferromagnetic (FM) ordering at a quantum critical point (QCP) [27]. Such novel critical phenomena are observed close to $T=0 \mathrm{~K}$. More recently, we have found very similar properties for $\mathrm{ThFe}_{2} \mathrm{Al}_{10}$ [28], and we suggest the presence of spin correlations and spin fluctuations (SFs) of Fe moments. In this orthorhombic structure, the $T$-atoms are stacked in twodimensional (2D) planes perpendicular to the $b$-axis. This layout means that SFs reside here mainly in the $a c$ plane, and the Sommerfeld coefficient $\gamma(0 \mathrm{~K})$ of $\mathrm{YFe}_{2} \mathrm{Al}_{10}$ is increased to $25 \mathrm{~mJ} \mathrm{~mol}^{-1} \mathrm{~K}^{-2}$ [29], while this value falls to $8.3 \mathrm{~mJ} \mathrm{~mol}{ }^{-1} \mathrm{~K}^{-2}$ for $\mathrm{YRu}_{2} \mathrm{Al}_{10}$ [30]. This observation and that for $\mathrm{LaFe}_{2} \mathrm{Al}_{10}$ [26] emphasize the special role of the $\mathrm{Fe}$ atoms in such cage-like ternaries. The Sommerfeld coefficient of $\mathrm{ThFe}_{2} \mathrm{Al}_{10}$ also has a similar value $\left(=22.5 \mathrm{~mJ} \mathrm{~mol}^{-1} \mathrm{~K}^{-2}\right)[28]$.

In view of the very interesting behaviors of the entire $\mathrm{Ce}_{2} \mathrm{Al}_{10}$ series, it has been important to compare them with those of the uranium isostructural series. The first synthesis of $\mathrm{UT}_{2} \mathrm{Al}_{10}(T=\mathrm{Fe}, \mathrm{Ru}$ and Os) single crystals and investigations of their X-ray and magnetic properties were carried out by Sugai et al. [31]. In contrast to the large number of rare-earth $R T_{2} \mathrm{Al}_{10}$ ternaries, which, despite showing large $R-R$ distances, do exhibit magnetic order at very low temperatures, all of these uranium systems lack any magnetic ordering, at least down to $2 \mathrm{~K}$ [31]. We previously carefully analyzed data obtained for single crystalline samples of $\mathrm{UFe}_{2} \mathrm{Al}_{10}$ [32]. We considered a scenario for this compound whereby the $5 f$ electrons of uranium display so-called dualism, i.e. two of them are localized, yielding a $\mathrm{U}^{4+}$ ion, and the remaining $5 f$ electron forms a broad metallic band with the $6 d$ and $7 s$ electrons. This electron configuration gives the unique possibility of treating $\mathrm{UFe}_{2} \mathrm{Al}_{10}$ as an ionic system in which the crystal field effect plays an important role. In fact, this effect is responsible for the strongly anisotropic behavior in the temperature dependence of the susceptibility in the paramagnetic region on the one hand, and simultaneously leads to its metallic character with an enhanced effective mass, on the other. This kind of behavior in the $\mathrm{UT}_{2} \mathrm{Al}_{10}$ species is similar to that observed for $\mathrm{UPd}_{3}$. This compound has been regarded for some time as a metallic system that nevertheless also shows the typical ionic properties of the $\mathrm{U}^{4+}$ ion [33 and references therein].

In this paper, we present the structure, thermodynamics and electron transport results obtained for single crystalline $\mathrm{URu}_{2} \mathrm{Al}_{10}$. We have previously published this bulk data for a polycrystalline sample of this compound [34]. We have also analyzed the electronic structure of this material in terms of a comparison to its Ce counterpart [35]. We are convinced that much wider future studies of the $\mathrm{UT}_{2} \mathrm{Al}_{10}$ systems will bring a great deal of valuable information on the dualism of $5 f$-electrons in actinide compounds [36].

\section{Experimental methods}

$\mathrm{ThRu}_{2} \mathrm{Al}_{10}$ and $\mathrm{URu}_{2} \mathrm{Al}_{10}$ single crystals were prepared using the molten aluminium flux technique, as described in [32]. The starting materials were Th (99.5\%), U (99.5\%), Ru (99.99\%) and $\mathrm{Al}(99.999 \%)$. 
The chemical composition, crystallographic quality and orientation of the obtained single crystals were verified both by scanning electron microscopy coupled with energy dispersive spectroscopy (SEM-EDS) and X-ray diffraction (XRD) techniques. No inclusion of an impurity phase was observed using either technique.

Single-crystal XRD was performed at room temperature (RT) to resolve the crystal structure, either on very small crystals from the flux (Th) or on a broken piece of a larger crystal (U). The diffraction intensities were collected at RT with a Nonius Kappa CCD four-circle diffractometer equipped with a bidimensional CCD detector using Mo $\mathrm{K}_{\alpha}$ radiation $(\lambda=0.71073 \AA)$. The integration and reduction of the redundant reflections of the different data sets, and the cell refinements, were performed using SADABS software [37]. Structural models were determined by direct methods using SIR-97 [38]. All structural refinements and Fourier syntheses were carried out with the help of SHELXL-97 [39]. The atomic positions were standardized using STRUCTURE TIDY [40]. Most of the crystals presented the $a$-axis perpendicular to the plate base.

The magnetic susceptibility of $\mathrm{URu}_{2} \mathrm{Al}_{10}$ between 2 and $300 \mathrm{~K}$ and the magnetization up to $9 \mathrm{~T}$ were measured using a Quantum Design MPMS-5 magnetometer. The specific heats of single crystalline samples of $\mathrm{ThRu}_{2} \mathrm{Al}_{10}$ and $\mathrm{URu}_{2} \mathrm{Al}_{10}$ with masses of $10 \mathrm{mg}$ and $4.0 \mathrm{mg}$, respectively, were measured using the thermal relaxation method in the temperature range 2-300 K using a Quantum Design PPMS platform. The samples were glued to the holder using Apiezon $\mathrm{N}$ vacuum grease addenda, the specific heat of which was first measured.

The electrical resistivity measurements of $\mathrm{URu}_{2} \mathrm{Al}_{10}$ were performed on single-crystalline samples in the form of bar-shaped specimens; these were cut from irregular plate-like single crystals, with dimensions of about $1.0 \times 0.5 \times 0.3 \mathrm{~mm}^{3}$ along the $a$ direction and $0.8 \times 0.25 \times 0.15 \mathrm{~mm}^{3}$ along the $c$ direction. Electrical contacts (four points) were made by the electrochemical deposition of $\mathrm{Cu}$, and finally, thin silver wires were glued using a silver paste. The measurements were carried out in a ${ }^{3} \mathrm{He}$ cryostat in the $0.3-300 \mathrm{~K}$ temperature range using an $\mathrm{AC}$ method. The magnetoresistivity was measured for the same samples in an applied magnetic field of up to $9 \mathrm{~T}$. In turn, a homemade setup [41] was used for measurements of the thermoelectric power of $\mathrm{URu}_{2} \mathrm{Al}_{10}$ at temperatures between 0.4 and $300 \mathrm{~K}$. The frontal surfaces of the same samples along the $a, b$ and $c$ directions were wetted with a liquid In-Ga alloy in order to improve the thermal and electrical contacts with the chamber plates.

\section{Results and discussion}

\subsection{Structure refinements}

The crystal-structure parameters for the ruthenium Th- and U-ternary aluminides obtained by single crystal XRD data refinement are listed in Table 1 . Data for $\mathrm{ThRu}_{2} \mathrm{Al}_{10}$ are given for the first time, while those for $\mathrm{URu}_{2} \mathrm{Al}_{10}$ are very close to the values given in the previous single-crystalline report [31] and slightly higher than those found in our first study [34]. The unit cell volume of $\mathrm{URu}_{2} \mathrm{Al}_{10}$ determined in these three studies is slightly reduced: 848.8(1), 845.1(1) and 843.4(1) $\AA^{3}$, respectively. Using these crystallographic data and those taken from [28], we can compare the lattice parameters of ruthenium and iron-based ternaries for both Th $(\mathrm{U})$ aluminides. The $\left[(a, b \text { or } c)_{\mathrm{Ru}}-(a, b\right.$ or $\left.c)_{\mathrm{Fe}}\right] \times 100 /(a, b \text { or } c)_{\mathrm{Fe}}$ ratios are $+0.79 \%(+0.66 \%),+0.30 \%(+0.11 \%)$ and $+0.92 \%(+0.67 \%)$ for the $a-, b$ - and $c$-axes, respectively. It can be inferred from these data that the magnitude of elongation in the $a c$-plane has an effect three (Th) to six (U) times larger than that along the $b$-axis. The influence of the larger Ru metallic radius $\left(\mathrm{r}_{\mathrm{Ru}}=1.345 \AA\right.$ [42]) than that of $\mathrm{Fe}\left(\mathrm{r}_{\mathrm{Fe}}=1.274 \AA\right.$ [42]) is thus highly anisotropic, suggesting stronger bonding along the $a$ - and $c$-axes as compared to the $b$-axis. Table 2 displays the atomic positions for ruthenium, Th- and U-aluminides, while Table 3 shows the selected interatomic distances for both Ru-ternaries. Of the five distinctive $\mathrm{Al}$ sites, the distances between $\mathrm{U}(\mathrm{Th})$ and the two $\mathrm{Al}(1)$, two $\mathrm{Al}(2)$, two $\mathrm{Al}(3)$ and four $\mathrm{Al}(4)$ are close to one another $\{\sim 3.15(5)$ [3.25(5)]\} $\AA$, whereas those for the four $\mathrm{Al}(5)$ and another two $\mathrm{Al}(1)$ atoms are larger. Details of the crystal structure are given in [43] and [44]. The distances between $\mathrm{Ru}$ and $\mathrm{Al}$ atoms are rather smaller than the sum of the corresponding metallic radii, and range between 2.6 and $2.8 \AA$. As mentioned previously in the case of $\mathrm{UFe}_{2} \mathrm{Al}_{10}$ [32], and below for $\mathrm{URu}_{2} \mathrm{Al}_{10}$, we cannot discuss the distances within $\mathrm{U}-\mathrm{Ru}(\mathrm{Al})$ using the framework of the sum of metallic radii (see [42]), as is often done in the literature. This is because in this kind of caged system, the uranium atoms should instead be treated as 
$\mathrm{U}^{4+}$-like ions experiencing crystal-field interactions. In this situation, the difference should be taken into account between a $\mathrm{U}^{4+}$-ion radius $(0.95 \AA)$ and a metallic one $(1.543 \AA)$ [42]. This means that the uranium ion in its large cage behaves as a free ion, but is under the influence of strong CF interactions. To get an idea of the positions of the $\mathrm{Al}$ atoms around the central uranium atom, we present the surroundings in Fig. 1; this image is viewed from the $b$-axis in one of the lower atomic layers of the unit cell formed by $\mathrm{U}, \mathrm{Ru}$ and $\mathrm{Al}(1)-\mathrm{Al}(4)$. This is also shown in Fig. 5(c) of [44] using dashed lines, and the positions of the particular $\mathrm{Al}$ atoms are marked by different colors.

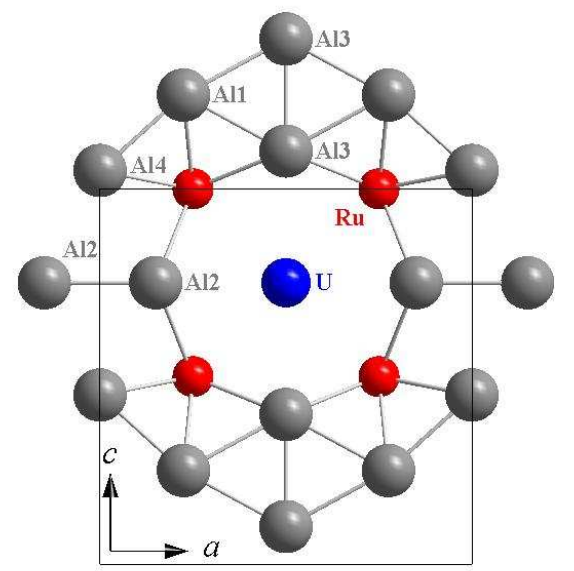

Fig. 1. Projection along the $b$-axis of the lower layer formed by $\mathrm{U}, \mathrm{Ru}$ and $\mathrm{Al}(1)-\mathrm{Al}(4)$ atoms [43]. This presents a two-dimensional system. Such layers are separated by the layers of the $\mathrm{Al}(5)$ atoms along the $b$-axis.

Table 1

Crystallographic data and structure refinement for $\mathrm{ThRu}_{2} \mathrm{Al}_{10}$ and $\mathrm{URu}_{2} \mathrm{Al}_{10}$.

\begin{tabular}{lll}
\hline Empirical formula & $\mathrm{ThRu}_{2} \mathrm{Al}_{10}$ & $\mathrm{URu}_{2} \mathrm{Al}_{10}$ \\
Formula weight $\left(\mathrm{g} \mathrm{mol}^{-1}\right)$ & 703.99 & 709.98 \\
Structure-type & $\mathrm{YbFe}_{2} \mathrm{Al}_{10}$ & $\mathrm{YbFe}_{2} \mathrm{Al}_{10}$ \\
Space group & $\mathrm{Cmcm}\left(\mathrm{n}^{\circ} 63\right)$ & $\mathrm{Cmcm}\left(\mathrm{n}^{\circ} 63\right)$ \\
Unit cell parameters $(\AA)$ & $a=9.088(1)$ & $a=9.040(1)$ \\
& $b=10.324(1)$ & $b=10.263(1)$ \\
& $c=9.200(1)$ & $c=9.149(1)$ \\
Unit cell volume $\left(\AA^{3}\right)$ & $863.2(2)$ & $848.8(1)$ \\
Z/calculated density $\left(\mathrm{g} \mathrm{cm}^{-3}\right)$ & $4 / 5.417$ & $4 / 5.556$ \\
Absorption coefficient $\left(\mathrm{mm}^{-1}\right)$ & 21.581 & 23.501 \\
Crystal colour and habit & metallic, prism & metallic, prism \\
Theta range & $3.72^{\circ}-41.99^{\circ}$ & $3.00^{\circ}-44.99^{\circ}$ \\
Limiting indices & $-17 \leq \mathrm{h} \leq 14$ & $-15 \leq \mathrm{h} \leq 17$ \\
& $-19 \leq \mathrm{k} \leq 11$ & $-20 \leq \mathrm{k} \leq 15$ \\
& $-17 \leq 1 \leq 16$ & $-18 \leq 1 \leq 17$ \\
Collected/unique reflections & $9646 / 1639$ & $13262 / 1901$ \\
$\mathrm{R}($ int $)$ & 0.0595 & 0.0513 \\
Absorption correction & $\mathrm{Semi}-\mathrm{empirical}$ & $\mathrm{Semi}-\mathrm{empirical}$ \\
Data/restraints/parameters & $1639 / 0 / 41$ & $1901 / 0 / 41$ \\
Goodness of fit on $\mathrm{F}^{2}$ & 1.125 & 1.083 \\
$\mathrm{R}$ indices [I>2 $\sigma(\mathrm{I})] \mathrm{a}$ & $\mathrm{R} 1=0.0283$ & $\mathrm{R} 1=0.0274$ \\
& $\mathrm{wR} 2=0.0609$ & $\mathrm{wR} 2=0.0388$ \\
Extinction coefficient & $0.0086(3)$ & $0.00180(7)$ \\
Largest difference peak and hole $\left(\mathrm{e} \AA^{-3}\right)$ & $8.383 /-6.308$ & $3.298 /-3.067$ \\
\hline
\end{tabular}


Table 2

Atomic parameters and equivalent displacement parameters for $\mathrm{ThRu}_{2} \mathrm{Al}_{10}$ and $\mathrm{URu}_{2} \mathrm{Al}_{10}$.

\begin{tabular}{llllll}
\hline Atoms & $\begin{array}{l}\text { Wyckoff } \\
\text { position }\end{array}$ & $\mathrm{x}$ & $\mathrm{y}$ & $\mathrm{z}$ & $\mathrm{U}_{\text {eq }}\left(\AA^{2}\right)$ \\
\hline $\mathrm{Th}$ & $4 c$ & 0 & $0.1277(1)$ & $1 / 4$ & $0.005(1)$ \\
$\mathrm{Ru}$ & $8 d$ & $1 / 4$ & $1 / 4$ & 0 & $0.004(1)$ \\
$\mathrm{Al} 1$ & $8 g$ & $0.2257(2)$ & $0.3672(1)$ & $1 / 4$ & $0.007(1)$ \\
$\mathrm{Al} 2$ & $8 g$ & $0.3523(1)$ & $0.1311(1)$ & $1 / 4$ & $0.007(1)$ \\
$\mathrm{Al} 3$ & $8 f$ & 0 & $0.1584(1)$ & $0.6019(2)$ & $0.007(1)$ \\
$\mathrm{Al} 4$ & $8 f$ & 0 & $0.3776(1)$ & $0.0478(2)$ & $0.006(1)$ \\
$\mathrm{Al} 5$ & $8 e$ & $0.2259(2)$ & 0 & 0 & $0.006(1)$ \\
& & & & & \\
$\mathrm{U}$ & $4 c$ & 0 & $0.1260(1)$ & $1 / 4$ & $0.006(1)$ \\
$\mathrm{Ru}$ & $8 d$ & $1 / 4$ & $1 / 4$ & 0 & $0.004(1)$ \\
$\mathrm{Al} 1$ & $8 g$ & $0.2243(1)$ & $0.3656(1)$ & $1 / 4$ & $0.007(1)$ \\
$\mathrm{Al} 2$ & $8 g$ & $0.3502(1)$ & $0.1304(1)$ & $1 / 4$ & $0.007(1)$ \\
$\mathrm{Al} 3$ & $8 f$ & 0 & $0.1556(1)$ & $0.6008(1)$ & $0.006(1)$ \\
$\mathrm{Al} 4$ & $8 f$ & 0 & $0.3763(1)$ & $0.0490(1)$ & $0.007(1)$ \\
$\mathrm{Al} 5$ & $8 e$ & $0.2238(1)$ & 0 & 0 & $0.007(1)$ \\
\hline
\end{tabular}

Table 3

Interatomic distances $(\AA)$ selected for $\mathrm{ThRu}_{2} \mathrm{Al}_{10}$ and $\mathrm{URu}_{2} \mathrm{Al}_{10}$.

\begin{tabular}{|c|c|c|c|c|c|}
\hline \multirow[t]{8}{*}{ Th } & $-2 \mathrm{Al} 4$ & $3.180(2)$ & \multirow[t]{8}{*}{$\mathrm{Ru}$} & $-2 \mathrm{Al} 5$ & \multirow{2}{*}{2.5903 (3) } \\
\hline & $-2 \mathrm{Al} 2$ & $3.202(2)$ & & -2 All & \\
\hline & - 2 Al1 & $3.212(2)$ & & $-2 \mathrm{Al} 3$ & $2.6334(7)$ \\
\hline & $-2 \mathrm{Al} 3$ & $3.252(2)$ & & $-2 \mathrm{Al} 4$ & $2.6630(7)$ \\
\hline & $-2 \mathrm{Al} 3$ & $3.253(2)$ & & $-2 \mathrm{Al} 2$ & $2.7679(8)$ \\
\hline & -4 Al5 & $3.353(1)$ & & & \\
\hline & $-4 \mathrm{Ru}$ & $3.4706(3)$ & & & \\
\hline & -2 Al1 & $3.6673(3)$ & & & \\
\hline U & $-2 \mathrm{Al} 4$ & $3.152(2)$ & $\mathrm{Ru}$ & $-2 \mathrm{Al} 5$ & $2.572(2)$ \\
\hline & $-2 \mathrm{Al} 2$ & $3.162(2)$ & & -2 Al1 & $2.580(2)$ \\
\hline & -2 Al1 & $3.182(2)$ & & $-2 \mathrm{Al} 3$ & $2.621(2)$ \\
\hline & $-2 \mathrm{Al} 3$ & $3.190(2)$ & & $-2 \mathrm{Al} 4$ & $2.640(2)$ \\
\hline & $-2 \mathrm{Al} 3$ & $3.213(2)$ & & $-2 \mathrm{Al} 2$ & $2.742(2)$ \\
\hline & $-4 \mathrm{Al} 5$ & $3.309(2)$ & & & \\
\hline & $-4 \mathrm{Ru}$ & $3.45(1)$ & & & \\
\hline & -2 Al1 & $3.648(9)$ & & & \\
\hline
\end{tabular}

\subsection{Electronic structure}

We have already calculated the electronic structure of isostructural $\mathrm{ThFe}_{2} \mathrm{Al}_{10}$ [28], $\mathrm{UFe}_{2} \mathrm{Al}_{10}$ [32] and (U;Ce) $\mathrm{Ru}_{2} \mathrm{Al}_{10}$ [35]. Therefore, in order to complete these data, we present the results of the calculations carried out for $\mathrm{ThRu}_{2} \mathrm{Al}_{10}$. These calculations are based on a fully relativistic version of the full-potential local-orbital (FPLO) method [45] and a local density approximation (LDA) of the exchange-correlation potential [46]. The obtained results allow us to draw some general conclusions about the whole group of these cage-type ternaries. The methods used in our calculations and selected $\boldsymbol{k}$-point mesh size $(12 \times 12 \times 12)$ in the Brillouin zone $(\mathrm{BZ})$ are the same as those described in [28]. As previously, we also assumed our experimental lattice parameters and the atomic positions given above to determine the band structure, total and partial densities of states (DOS) per formula unit (f.u.) and the Fermi surface (FS) of $\mathrm{ThRu}_{2} \mathrm{Al}_{10}$.

The total and partial DOS of $\mathrm{ThRu}_{2} \mathrm{Al}_{10}$ are presented in Fig. 2(a). As is apparent from this figure, all the constituent atoms of $\mathrm{ThRu}_{2} \mathrm{Al}_{10}$, forming the atomic cage in a $\mathrm{YbFe}_{2} \mathrm{Al}_{10}$-type crystal 
structure have remarkable contributions to DOS at the Fermi level $\left(E_{\mathrm{F}}\right)$, as in the case of the isostructural reference systems mentioned above. Furthermore, the valley of DOS cuts $E_{\mathrm{F}}$, yielding a moderately low value of the Sommerfeld coefficient $\gamma_{6}=6.2 \mathrm{~mJ} \mathrm{~mol}^{-1} \mathrm{~K}^{-2}$, which is typical of normal metallic systems and in good agreement with our experimentally estimated value $\left(7.6 \mathrm{~mJ} \mathrm{~mol}^{-1} \mathrm{~K}^{-2}\right)$. This comparison of the experimental $\chi 0)$ and theoretical $\gamma_{6}$ values indicates rather weak electronelectron correlations. It stands in contradiction to the case of the $\mathrm{ThFe}_{2} \mathrm{Al}_{10}$ counterpart, where the slightly higher value of the calculated Sommerfeld coefficient $\left(7.8 \mathrm{~mJ} \mathrm{~mol}^{-1} \mathrm{~K}^{-2}\right)$ is substantially lower than the experimental one $\left(25(1) \mathrm{mJ} \mathrm{mol}^{-1} \mathrm{~K}^{-2}\right)$ [28]. The DOS around $E_{\mathrm{F}}$ is dominated by $\mathrm{Al} 3 s p d$ electrons, coming from all five atomic sites in the unit cell. In turn, the almost equal contributions from the hybridized Th $6 d-5 f$ states and the $\mathrm{Ru} 4 d$ contributions are both half as large as the $\mathrm{Al}$ ones; this is unlike the situation taking place in the $\mathrm{ThFe}_{2} \mathrm{Al}_{10}$ analogue, where the $\mathrm{Fe} 3 d$ contribution is a little higher than that of the $\mathrm{Ru} 4 d$ electrons and is equal to that obtained for the $\mathrm{Al}$ electron contribution (see the inset in Fig. 2(a)). Simultaneously, the $\mathrm{U} 5$ contribution of $\mathrm{URu}_{2} \mathrm{Al}_{10}$ was much higher than the others, and yielded a relatively large calculated $\gamma_{\mathrm{b}}$-value $\left(21.5 \mathrm{~mJ} \mathrm{~mol}^{-1} \mathrm{~K}^{-2}\right.$ [35]) which is in good agreement with the experimental $\gamma(0)$-value $\left(22.0 \mathrm{~mJ} \mathrm{~mol}^{-1} \mathrm{~K}^{-2}\right)$ (see below).
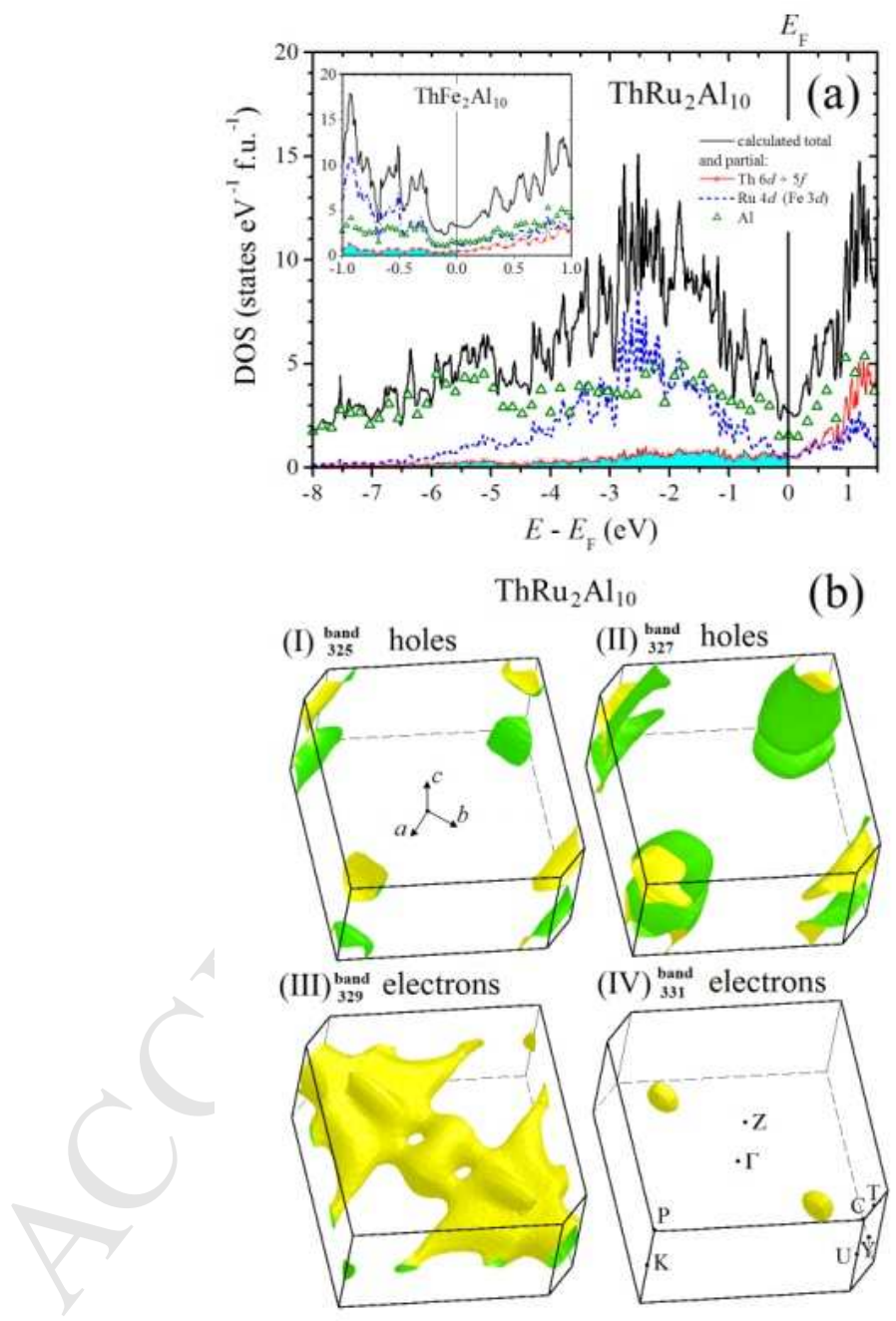

Fig. 2. (a) Fully relativistic (LDA) total (solid line) and partial DOS calculated for $\mathrm{ThRu}_{2} \mathrm{Al}_{10}$ compared to DOS of $\mathrm{ThFe}_{2} \mathrm{Al}_{10}$ near $E_{\mathrm{F}}$ (inset based on our results in Ref. [28]). The cyan (hatched) area denotes contributions of primarily occupied Th $6 d$ states within the hybridized Th $6 d-5 f$ contribution. (b) The corresponding FS sheets of $\mathrm{ThRu}_{2} \mathrm{Al}_{10}$ originating from four Kramers double-degenerate bands (Nos. 325, 327, 329, 331), drawn separately within the orthorhombic BZ boundaries. Dark (green) and light (yellow) colors reflects the inside (electrons) and outside (holes) of FS, respectively. 
In $\mathrm{ThRu}_{2} \mathrm{Al}_{10}$, the thorium contribution to the hybridized $\mathrm{Th} 6 d-5 f$ states mentioned above forms a long tail far below $E_{\mathrm{F}}$ (see the hatched area in Fig. 2(a)); however, above $E_{\mathrm{F}}$, unoccupied $5 f$ states start to dominate over the $6 d$ ones. In turn, the Al 3spd electrons yield a broad structure in the whole energy region, as shown in Fig. 2(a). Finally, the Ru $4 d$ electrons create a distinctly multipeak structure, with a maximum located at around $-2.5 \mathrm{eV}$, shifted further below $E_{\mathrm{F}}$ by about $1 \mathrm{eV}$ with respect to that of the $\mathrm{Fe} 3 d$ electrons in $\mathrm{ThFe}_{2} \mathrm{Al}_{10}$. It is worth highlighting that the DOS coming from $\mathrm{Ru}$ and all the $\mathrm{Al}$ atomic positions in $\mathrm{ThRu}_{2} \mathrm{Al}_{10}$ are comparable to those of $\mathrm{URu}_{2} \mathrm{Al}_{10}$ [35] in the entire energy region considered. This shows that the band structure originating from the $\left[\mathrm{Ru}_{2} \mathrm{Al}_{10}\right]$ assembly is nearly the same in both aluminides (see the inset in Fig. 2(a)).

Interestingly, the $\mathrm{FS}$ of $\mathrm{ThRu}_{2} \mathrm{Al}_{10}$ computed here and shown in Fig. 2(b) is identical to that of $\mathrm{ThFe}_{2} \mathrm{Al}_{10}$ [28], despite some differences in the DOS around $E_{\mathrm{F}}$, as discussed above. The FSs of both systems consist of as many as four sheets originating from Kramers double-degenerate bands. The sheets are derived from two lower bands (325th and 327th) comprising three-dimensional hole-like closed pockets of rather small size, located in the corners of the BZ. At the same time, the other two sheets possess an electron-like character. They contain a large, flattened open structure (329th band), which is strongly anisotropic in the $c$ direction versus the $a b$ plane, probably due to substantial hybridization anisotropy. There are also small closed pockets (331th band). On the one hand, this type of FS suggests distinctly similar metallic behaviour for the Th-based aluminides; however, on the other, the FS sheets have shapes that are quite different from those of $\mathrm{URu}_{2} \mathrm{Al}_{10}$ [35], whose $\mathrm{U} 5 f$ electrons contribute only partly to the FS. Interestingly, the FS sheets of $\mathrm{ThRu}_{2} \mathrm{Al}_{10}$ resemble those of isostructural $\mathrm{CeRu}_{2} \mathrm{Al}_{10}$ [35], except for the lack of the corresponding fourth sheet (with the small electron pockets) in the latter compound, although the DOS of both these systems around $E_{\mathrm{F}}$ are quite different. This relation of FSs is analogous to that existing between, for example, $\operatorname{ThRhIn}_{5}$ and

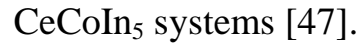

Based on our partial DOS results, we also estimate for $\mathrm{ThRu}_{2} \mathrm{Al}_{10}$ the overall magnitude of the temperature dependence of thermoelectric power (TEP), $S_{\mathrm{d}}(T)$, using the Mott model for diffusion TEP, employing the method given in Eq. (14) in [28] for $\mathrm{ThFe}_{2} \mathrm{Al}_{10}$. The absolute value of the slope obtained for $\mathrm{ThRu}_{2} \mathrm{Al}_{10}, S_{\mathrm{d}} T^{1}=-0.0060 \mu \mathrm{V} \mathrm{K} \mathrm{K}^{-2}$, is much lower than that obtained for the iron analogue $\left(-0.0197 \mu \mathrm{V} \mathrm{K}^{-2}[28]\right)$.

\subsection{Magnetic properties}

\subsubsection{Experimental magnetic susceptibility}

Fig. 3(a) shows the temperature-dependent magnetic susceptibility, $\chi_{i}(T)$, measured along the three crystallographic directions $(i=a, b, c)$ of an $\mathrm{URu}_{2} \mathrm{Al}_{10}$ single crystal. This dependence exhibits strong magnetic anisotropy, for which the easy magnetization axis is the $c$-axis, i.e. $\chi_{c}>\chi_{a}>\chi_{b}$. This finding is in accordance with the previous report by Sugai et al. [31]; however, it is different from that reported by us for $\mathrm{UFe}_{2} \mathrm{Al}_{10}$ in [32], where the $a$-axis was found to be the easy one, with $\chi_{a}>\chi_{c}>\chi_{b}$. Similarly, the $\chi_{i}(T)$ of $\mathrm{CeFe}_{2} \mathrm{Al}_{10}$ [48] and $\mathrm{CeRu}_{2} \mathrm{Al}_{10}$ [49] also exhibit magnetic anisotropy, and the susceptibility along the $a$-axis has the highest values. Interestingly, all the examples discussed above show not only the smallest susceptibility values along the $b$-axis but also a weak temperature variation of the latter below RT. In addition, our measurements taken along this axis yield a very broad susceptibility maximum at around $200 \mathrm{~K}$. In all the above mentioned cases, it is clear that susceptibilities below about $100 \mathrm{~K}$ reveal a tendency to saturation, which however is followed by a small upturn at the lowest temperatures. Similar behavior of the susceptibility at low temperatures was also reported by Sugai et al. [31]. We believe that this upturn most likely arises from impurities, and therefore the correct low-temperature dependencies of our susceptibility curves are determined by subtracting $\chi_{\text {imp }}$ (assuming this follows a $C / T$ Curie law) from the respective experimental values. Finally we obtain approximately constant $\chi_{i}(0)$ saturated values for all three directions at $T=0 \mathrm{~K}$. As an example, we present in Fig. 3(a) a correction procedure for the susceptibility using thick dashed lines which generally coincide with the theoretical solid lines.

Fig. 4 indicates the field-dependent magnetization, $M(\mathrm{~B})$, taken at $2 \mathrm{~K}$, yields along the $a$-axis a perfect straight line, while for the remaining axes $M(\mathrm{~B})$ may be either approximated by a very weakly marked curvature, probably arising from a negligibly small magnetic correlation, or a small deviation within an experimental error. Nevertheless, we do not observe any influence on $M(\mathrm{~B})$ due to 
ferromagnetic impurities. This allows us to infer that the susceptibilities approach limited values at $T=$ $0 \mathrm{~K}$. Thus, the inverse values of these corrected susceptibilities are plotted in Fig. 3(b) as a function of temperature. The Curie-Weiss (CW) behavior for the three axes is marked in Fig. 3(b) by thin dashed lines. The corresponding magnetic parameters are listed in Table 4. Note that the large negative paramagnetic Curie temperatures, $\theta_{\mathrm{p}}$, do not reflect reality, especially those determined along the $b$ axis. It is clear that in these situations, the measurements need to be made at much higher temperature range. We then applied the crystal field (CF) theory in order to explain the observed highly anisotropic behavior of the susceptibility of $\mathrm{URu}_{2} \mathrm{Al}_{10}$. As is well known in the case of $5 f$-electron systems, this theory should be based on an equal footing of the intra-atomic interactions, i.e. Coulombic, spinorbital and crystal-field interactions.

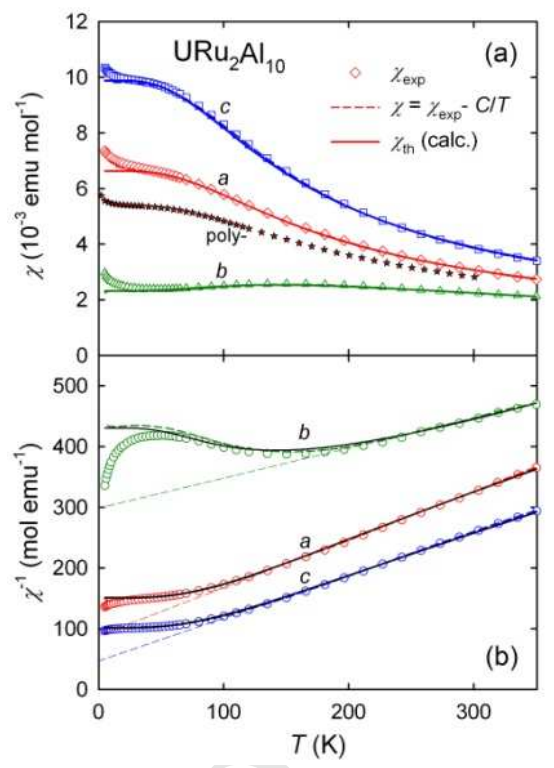

Fig. 3. (a) Temperature dependencies of the magnetic susceptibility measured along the three main crystallographic directions of orthorhombic $\mathrm{URu}_{2} \mathrm{Al}_{10}$. Low-temperature small upturn in $\chi_{\mathrm{i}}(T)$ (where $i=a, b, c$ ) is assumed to come from impurities, the susceptibilities of which follow the Curie law. Examples of their correction is presented in the figure by respective dashed curves which, however, cover the solid lines reflecting $\mathrm{CF}$ calculations. In addition we show the susceptibility variation determined on the polycrystalline sample in our previous paper [34]. (b) Corrected inverse susceptibilities versus temperature (symbols) compared to those calculated (see text), marked by solid curves. Calculations based on the CF splitting of ground multiplet (see in Fig. $8 \mathrm{CF}$ scheme of levels in $\mathrm{K}$ units), resulted for a $5 f^{2}$-electron configuration of $\mathrm{U}^{4+}$ ion in here considered uranium-ruthenium-aluminide. The dashed lines correspond to the Curie-Weiss fitting with parameters presented in Table 4.

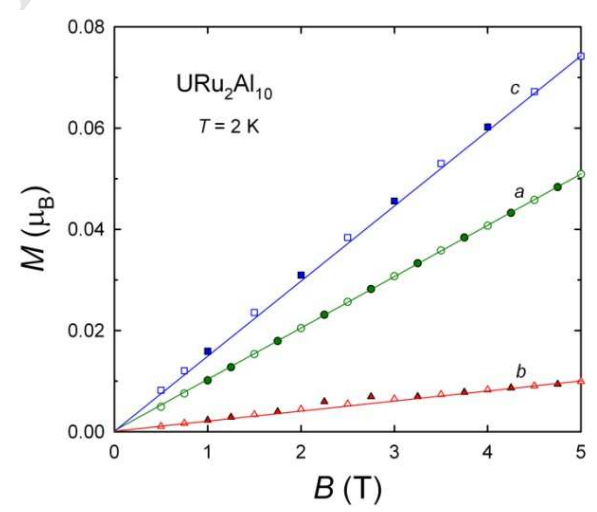

Fig. 4. $\mathrm{URu}_{2} \mathrm{Al}_{10}$ : magnetization $M$ versus magnetic field $B$ isotherms at $2 \mathrm{~K}$ measured along the three crystallographic directions. 
Table 4

Magnetic parameters of $\mathrm{UFe}_{2} \mathrm{Al}_{10}$ and $\mathrm{URu}_{2} \mathrm{Al}_{10}$ obtained by Curie-Weiss fitting of the high temperature region of the $\chi^{-1}(T)$ curves (Fig. 3(b)).

\begin{tabular}{lllll}
\hline Compound & axis & $\begin{array}{l}\chi_{\mathrm{M}}{ }^{300 \mathrm{~K}} \\
\left(10^{-3} \mathrm{emu}\right. \\
\left.\mathrm{mol}^{-1}\right)\end{array}$ & $\begin{array}{l}\theta_{\mathrm{p}} \\
(\mathrm{K})\end{array}$ & $\begin{array}{l}\mu_{\mathrm{eff}}{ }^{a} \\
\left(\mu_{\mathrm{B}}\right)\end{array}$ \\
\hline $\mathrm{UFe}_{2} \mathrm{Al}_{10}[32]$ & $a$ & 3.5 & -45 & 3.80 \\
& $b$ & 2.3 & -700 & $4.3^{*}$ \\
& $c$ & 3.1 & -160 & 3.38 \\
$\mathrm{URu}_{2} \mathrm{Al}_{10}$ & $a$ & 3.2 & -109 & 3.18 \\
& $b$ & 2.2 & -595 & $4.0^{*}$ \\
& $c$ & 3.9 & -62 & 3.35 \\
\hline
\end{tabular}

* Any meaning of $\mu_{\text {eff }}$ requires measurements performed at considerably higher temperatures.

\subsubsection{The crystal field model}

The strongly anisotropic magnetization characteristics of the $\mathrm{UT}_{2} \mathrm{Al}_{10}$ series confirm the presence of $5 f$-localized electrons in these intermetallic systems. Of the three hypothetical configurations expected for the uranium atom, i.e. $5 f^{1}, 5 f^{2}$ and $5 f^{3}$, only those with an even number of electrons admit finite values of the magnetic susceptibility at low temperatures (as shown in Fig. 3 for $\left.\mathrm{URu}_{2} \mathrm{Al}_{10}\right)$. The XPS data and DFT calculations [35] locate the ground multiplet formed by the $5 f^{2}$ electron configuration sufficiently below the Fermi energy to accept the conventional Hamiltonian for localized $f$-electrons, known for non-metallic systems [50]. Furthermore, for the same reason, the onsite Coulomb repulsion and the spin-orbit correction represented by the Slater integrals $F^{k}(k=2,4,6)$ and the spin-orbit coupling coefficient $\zeta_{5 f}$ should not diverge by a large amount. Hence, we may accept the values known for $\mathrm{UGeO}_{4}: F^{2}=61376 \mathrm{~K}, F^{4}=56803 \mathrm{~K}, F^{6}=35176 \mathrm{~K}, \zeta_{5 f}=2482 \mathrm{~K}$ [51]. Attention should be paid to the importance of the so-called " $J$-mixing" term in the case of the uranium compounds; this is still ignored in the literature, despite its strong influence on the sequence of the energy levels [52]. Simultaneous diagonalization of the "free-ion" interactions (Coulomb and spinorbit interactions with the crystal field) breaking the spherical symmetry solves the problem.

Thus, to get the eigenenergies $E_{v}$ and eigenvectors $\mid v>$ of the subsystem of localized electrons, we use the effective phenomenological Hamiltonian:

$$
H=\sum_{k} F^{k} f_{k}+\sum_{i} \zeta_{5 f} l_{i} \cdot s_{i}+\sum_{i} \sum_{k, q} B_{k q} C_{q}^{(k)}\left(r_{i} / r_{i}\right)+\mu_{B}\left(l_{i}+s_{i}\right) B
$$

where $i$ numbers the electrons; $k=2,4,6 ; \boldsymbol{f}_{k} \boldsymbol{s}, \boldsymbol{l}$ are the angular Coulomb, spin and orbit operators in the space spanned by the two-electron wave-functions; and $F^{k}, \zeta_{5 f}$ are the corresponding parameters which are kept constant here. The third quantity in Eq. (1) represents the crystal-field expansion in terms of the normalized spherical harmonics, where $C_{q}^{(k)}$ and $B_{k q}$ are the coefficients. For the actual $\mathrm{C}_{2 \mathrm{v}}$ symmetry of the uranium site, there are nine independent $B_{k q}$ parameters. In the case of the coordination system shown in Fig. $5, B_{k q}$ are real and non-zero only for $\mathrm{q} \geq 0(\mathrm{q} \leq \mathrm{k})$ and even. The last term represents the Zeeman energy in the magnetic field $\boldsymbol{B}$. Having obtained the eigenstates of the Hamiltonian in Eq. (1), the molar magnetization in direction $i$ is given by the formula:

$$
M_{m o l, i}=N_{A} \frac{\sum \mu_{v, i} \exp \left(-\beta E_{v, i}\right)}{\sum \exp \left(-\beta E_{v, i}\right)},
$$

where

$$
\mu_{v, i}=\frac{-\partial E_{v, i}}{\partial B_{i}}
$$

The corresponding molar susceptibility can be simply calculated by dividing the magnetization by external magnetic field: 


$$
\chi_{m o l, i}=\frac{M_{m o l, i}}{B_{i}}
$$

The measured temperature dependencies of the three components $i$ along the $a, b$ and $c$ crystallographic directions of the magnetic susceptibility can be modeled by fitting the $B_{k q}$ parameters of the Hamiltonian in Eq. (1). Because there are nine of these parameters, a relatively large number for the $C_{2 v}$ point symmetry, we firstly employ a simplified CF model called the angular overlap model $(\mathrm{AOM})$, according to which the CF parameters are linear combinations of so-called "intrinsic" parameters $\mathrm{e}_{\mu}^{\mathrm{t}}, \mu=0(\sigma), 1(\pi), 2(\delta) ; t=\mathrm{Ru}, \mathrm{Al}$. AOM was initially formulated for optical materials (see [53] and references therein) and was extended to metallic systems [54], with quantitative confirmation later found in microscopic theory [55]. Details of the application of the model to the present coordination sphere of the uranium atom can be found in [32].
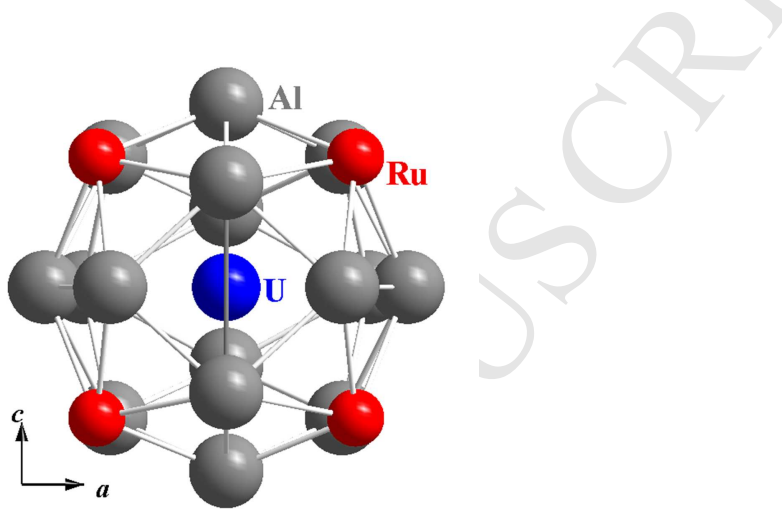

Fig. 5. An uranium central atom and its 20 nearest neighbors in $\mathrm{URu}_{2} \mathrm{Al}_{10}$ projected on the ac plane according to the crystallographic data from Table 2.

The AOM and CF parameters were first fitted directly to the experimental susceptibility data in the three $i$ directions as a function of temperature, using the Condon routine [56,57] adapted to the AOM approach using a version previously used by the authors [58]. The subsequent refined phase included the standardization of the parameters necessary to avoid their scattering between the equivalent settings of the nominal coordinate systems during fitting (see [59] and [32] for details).

The final set of the $B_{k q}$ parameters is compared in Table 5 to those from the AOM estimation and the previous results obtained for $\mathrm{UFe}_{2} \mathrm{Al}_{10}$ [32]. The $\mathrm{AOM}$ parameters for $\mathrm{URu}_{2} \mathrm{Al}_{10}$, from which the AOM set of the $B_{k q}$ parameters was evaluated, are the following (in K): large and negative $e_{\sigma}=$ $-1039,-2561$, positive $e_{\pi}=1980,1107$ and relatively small and positive $e_{\delta}=324,64$, for $\mathrm{U}-\mathrm{Al}$ and U$\mathrm{Ru}$ linear ligators, respectively. These values are similar to those for $\mathrm{UFe}_{2} \mathrm{Al}_{10}$, as reported earlier. The negative values of some of the intrinsic parameters require comment; the effective crystal field seen by the open shell electrons immersed in the sea of the conduction electrons is determined by purely quantum mechanisms, whereby the renormalization term arising from the hybridization of the localized states with the band states plays the main role [55]. This contribution is consistent with the AOM constraints and provides positive intrinsic parameters, just as the simple point charge model. However, there are other renormalization terms, some of which produce contributions opposite in sign to that of the hybridization contribution. The most important of these appears as a result of formation of the virtual bond state (VBS, see [60]). Its remarkable influence was discussed for the first time by Christodoulos et al. [61] for rare earths in noble metals, where the VBS was formed by $5 d$ electrons. For the systems under consideration, the VBS is formed by both the $6 d$ and $5 f$ electrons according to the postulated dual nature of the $5 f$ electrons, and the effect should therefore be enhanced. Although the VBS contribution does not fulfill the superposition requirement of the AOM approach, it may be partly reflected by the intrinsic parameters. Thus, the negative values of some of these may be regarded as a manifestation of the dual nature of the $5 f$ electrons. Of course, we expect that further studies at the microscopic level will confirm this reasoning. 
As for $\mathrm{UFe}_{2} \mathrm{Al}_{10}$, the refined values of $B_{k q}$ (Table 5) differ to some extent from their $\mathrm{AOM}$ estimates; this indicates the limited accuracy of the AOM approximation mentioned above. They are relatively large compared to the non-metallic uranium compounds (e.g. for $\mathrm{UO}_{2}$ [62]), although slightly smaller than those obtained for the iron analogue. This can clearly be seen from the energy level schemes presented in Fig. 8. Proximity to the Fermi energy enhances renormalization, due to the hybridization responsible for the $\mathrm{CF}$ effect in metals, and this has already been observed in nonperturbative treatments for $\mathrm{CeRh}_{3} \mathrm{Si}_{2}, \mathrm{UGe}_{2}$ and $\mathrm{UCu}_{2} \mathrm{Si}_{2}$ [63-65]. As discussed previously, despite obtaining relatively high absolute values of the $\mathrm{CF}$ parameters, the overall splitting of the ground multiplet (predominantly consisting of the ${ }^{3} \mathrm{H}_{4}$ state) is rather moderate $(2081 \mathrm{~K}$ ). It is likely that a mutual reduction of the fourth and sixth ranks also takes place in the CF potential of the eigenenergies.

\section{Table 5}

$\mathrm{AOM}$ and the refined sets of the parameters $B_{k q}$ (in $\mathrm{K}$ ) obtained with and without the AOM constraints as described in the main text for $\mathrm{URu}_{2} \mathrm{Al}_{10}$ and their comparison with the result for $\mathrm{UFe}_{2} \mathrm{Al}_{10}$ [32].

\begin{tabular}{lllll}
\hline \multicolumn{3}{c}{$\mathrm{UFe}_{2} \mathrm{Al}_{10}[32]$} & \multicolumn{2}{l}{$\mathrm{URu}_{2} \mathrm{Al}_{10}$} \\
$B_{k q}$ & $\mathrm{AOM}$ & Refined & $\mathrm{AOM}$ & Refined \\
\hline$B_{20}$ & 317 & -561 & 731 & 1000 \\
$B_{22}$ & -557 & -304 & -609 & 156 \\
$B_{40}$ & 5428 & 2977 & 4879 & 934 \\
$B_{42}$ & -2216 & -3064 & -2316 & -2700 \\
$B_{44}$ & -219 & -1695 & 29 & 67 \\
$B_{60}$ & -8097 & -11179 & -8572 & -5309 \\
$B_{62}$ & -7959 & -1089 & -8255 & -9595 \\
$B_{64}$ & 7425 & 8352 & 7001 & 8539 \\
$\mathrm{~B}_{66}$ & 1737 & -8470 & 748 & -2575 \\
\hline
\end{tabular}

As Fig. 3 indicates, our CF model fairly accurately describes the susceptibility measurements across the entire temperature range except for the lowest region, where the observed susceptibility tail can be attributed to undefined paramagnetic impurities. We notice that the model susceptibility curves are sensitive to the CF parameters, which enhances the reliability of the obtained results. A similar sensitivity is seen for $\mathrm{CeRu}_{2} \mathrm{Al}_{10}$ in [23], where the two sets of CF parameters GS\#1 and GS\#2 give almost the same energy level schemes, according to the edges of the X-ray absorption observed in $\mathrm{M}_{4,5}$, but completely different temperature dependencies of the magnetic susceptibility. The question is whether the two sets of parameters giving almost the same energies are really different. In fact, they become similar if they are expressed using the same coordinate system. Following the standardization described in [59], which in this case relies on the rotation of the nominal coordinate system of the set GS\#1 by Euler angles $(0, \pi / 2,0)$, the first set GS\#1 in Table 5 of [23], converted to the Wybourne normalization used here, becomes (in K): $B_{20}=488, B_{22}=237, B_{40}=-83, B_{42}=-1208, B_{44}=337$. This set is closer to GS\#2 than the original set GS\#1. More specifically, the closeness factor [59] of the two sets, the above rotated GS\#1 set and the original GS\#2 set, increased to 0.869 from the initial value of 0.241 . Comparing the GS\#1 set, regarded by the authors of [23] to be more reliable than GS\#2, with the parameters in Table 5 obtained for uranium for $\mathrm{k} \leq 4$, we see that these are smaller, as expected for the $4 f$ electron system with respect to the $5 f$ one. The extremely large six-rank parameters seen in our Table 5 indicate once again the participation of the band component of the $5 f$ electrons in the VBS, thus confirming their dual nature.

\subsection{Thermal properties}

The specific heat data $C_{\mathrm{p}}(T)$ of $\mathrm{URu}_{2} \mathrm{Al}_{10}$ and the reference compound $\mathrm{ThRu}_{2} \mathrm{Al}_{10}$, both measured between 2 and $300 \mathrm{~K}$ for single crystals, are shown in Fig. 6. As this figure illustrates, these curves lack any type of anomaly associated with any ordering, throughout the entire temperature range measured. The electronic specific heat constants, $\gamma(0)$, and the Debye temperatures, $\Theta_{\mathrm{D}}$, were found for $B=0$ in the usual way from a least-squares fit of the $C_{\mathrm{p}} / T$ vs. $T^{2}$ data, as shown in the upper inset in Fig. 6. There is an apparent difference from the variation of $C_{\mathrm{p}} / T$ measured in the case of 
(U;Th) $\mathrm{Fe}_{2} \mathrm{Al}_{10}$ [28]. For the latter ternaries, these data follow a curve including the magneticfluctuation term $T^{2} \ln T$, which exhibits an upturn in $C_{\mathrm{p}} / T$ at low temperatures. However, the curve behaves differently for the Ru-containing $\mathrm{U}$ - and Th-based aluminides; their $C_{\mathrm{p}} / T$ vs. $T^{2}$ functions are linear, and extrapolation to $T=0 \mathrm{~K}$ yields $\gamma(0) \approx 21.0(5)$ and $7.6(5) \mathrm{mJ} \mathrm{mol}^{-1} \mathrm{~K}^{-2}$, respectively. These values can be compared with the values of 28.5(1) and 22.5(5) $\mathrm{mJ} \mathrm{mol}^{-1} \mathrm{~K}^{-2}$ found for the corresponding Fe-containing aluminides [28]. It is clear that the $\gamma(0)$ value for $\operatorname{ThFe}_{2} \mathrm{Al}_{10}$, which is a result of the magnetic fluctuations at the iron site at low temperatures, is almost three times larger than that for $\mathrm{ThRu}_{2} \mathrm{Al}_{10}$. This may indicate the lack of magnetic fluctuations after an exchange of $\mathrm{Fe}$ by $\mathrm{Ru}$. Furthermore, the almost parallel behavior of the straight lines of both $C_{\mathrm{p}} / T$ vs. $T^{2}$ functions yields close values of $\Theta_{\mathrm{D}}$, which are roughly equal to $468(10) \mathrm{K}$. For ternaries containing Fe, $\Theta_{\mathrm{D}}=440(10) \mathrm{K}$ [28]. This is a consequence of the lattice contributions, which are almost the same for all these ternaries; these phases not only have the same crystal structure type with negligibly different lattice parameters, but also the same 4+ valence, and the difference in their molecular mass for each pair (U,Th) $\mathrm{Fe}_{2} \mathrm{Al}_{10}$ and $(\mathrm{U}, \mathrm{Th}) \mathrm{Ru}_{2} \mathrm{Al}_{10}$ is only about $0.9 \%$.

In the lower inset of Fig. 6, we plot the magnetic part of the specific heat, which is obtained by subtracting the specific heat of $\mathrm{ThRu}_{2} \mathrm{Al}_{10}$ from that of $\mathrm{URu}_{2} \mathrm{Al}_{10}$. The excess $\Delta C_{\mathrm{p}}(T)$ found from this subtraction is roughly treated as a Schottky-like contribution of the $5 f$-electrons. As shown in this lower inset, the solid curve is drawn as an average of the data. The obtained smooth curve shows a broad maximum at about $100 \mathrm{~K}$. This $C_{5 f}(T)$ contribution to the total specific heat $C_{\mathrm{p}}(T)$ is discussed later in this paper. The specific heat excess due to the magnetic contribution, however, is very tiny in comparison to the total specific heat values of the molecule, which contains as many as 13 atoms. This becomes especially important at higher temperatures, where this relative smallness leads to a considerable increase in the experimental error. Hence, we are able to obtain reasonable results only up to $250 \mathrm{~K}$. Any better determination of this excess would require much more precise measurements on heavier crystals, to decrease the deviation in the measured data. Despite these difficulties, the obtained Schottky-type anomaly is clear, indicating that the crystal field approach applied in the interpretation of the magnetic and transport properties of uranium aluminides containing $\mathrm{Fe}$ [28], $\mathrm{Ru}$ [present paper] and Os [in preparation] is fully justified. Hence, these $\mathrm{UT}_{2} \mathrm{Al}_{10}$ caged systems merit a much deeper study, as for $\mathrm{UPd}_{3}$. Indeed, this intermetallic compound was regarded for many years as a unique system, exhibiting $\mathrm{CF}$ interactions comparable to that acting in the insulator $\mathrm{UO}_{2}$ [33]. At present, there are few examples of uranium intermetallics for which crystal field excitations have been found (see discussion below).

As has recently been recognized (see Introduction), the phonon part of the caged-type compounds can be described by taking into account the Einstein modes (except for the Debye modes).

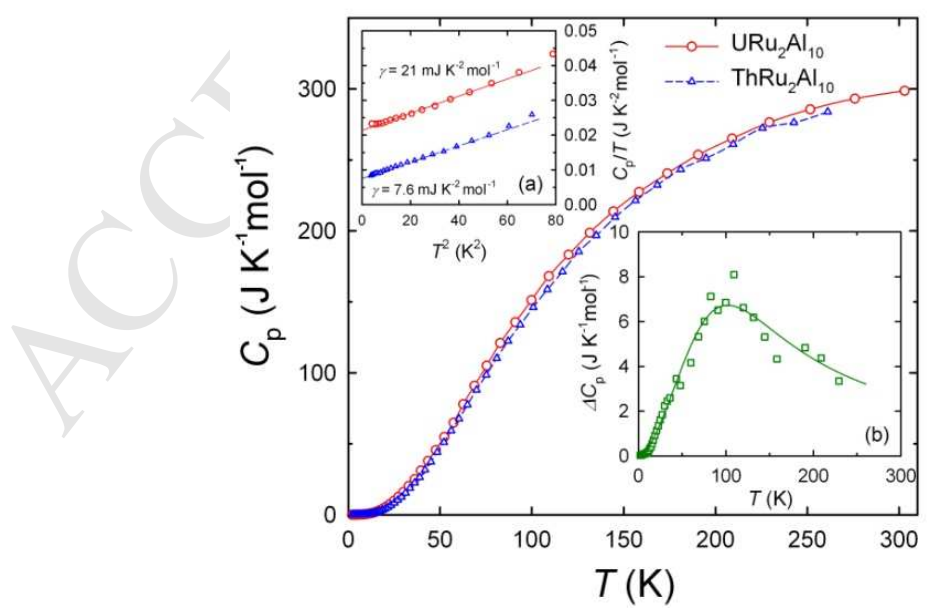

Fig. 6. The specific heat of $\mathrm{URu}_{2} \mathrm{Al}_{10}$ and $\mathrm{ThRu}_{2} \mathrm{Al}_{10}$ measured up to $\mathrm{RT}$. Inset (a) shows the corresponding $C_{\mathrm{p}} / T$ vs. $T^{2}$ curves, and inset (b) illustrates the experimental Schottky-type contribution to the specific heat of $\mathrm{URu}_{2} \mathrm{Al}_{10}$. The solid line is a smoothed average curve of $C_{5 f}(T)$. 
As pointed out in more detail previously [28], in view of this phonon behavior, the temperature variations of three components can be estimated: the Debye $C_{\mathrm{D}}(T)$, Einstein $C_{\mathrm{E}}(T)$ and electronic $C_{\mathrm{e}}(T)$ contributions. For this purpose, we first plot the $C_{\mathrm{p}}\left(\mathrm{ThRu}_{2} \mathrm{Al}_{10}\right) / T^{3}$ vs. $T$ dependence, together with the same curve for $\mathrm{URu}_{2} \mathrm{Al}_{10}$, as shown in Fig. 7. It should be recalled that the latter contains another contribution, namely the Schottky anomaly. As can be seen, this dependences for the Th- and U-based aluminides considered in this paper show maxima at $T_{\max }=22(1)$ and $19(1) \mathrm{K}$, respectively, indicating that we are concerned here with the optical modes except for the acoustic modes. Both the observed peaks are rather broad; these were discussed in a previous paper for (U;Th) $\mathrm{Fe}_{2} \mathrm{Al}_{10}$ [28]. We could estimate the main respective Einstein temperatures as $\Theta_{\mathrm{ETh}}(=110 \mathrm{~K})$ and $\Theta_{\mathrm{EU}}(=95 \mathrm{~K})$ based on the simple thermodynamic equation $\Theta_{\mathrm{E}} \approx 5 \times T_{\max }$. Of course, the observed broad peak in $C_{\mathrm{p}} / T^{3}$ vs. $T$ function can be deconvoluted into several peaks of lower intensity reflecting other $\Theta_{\mathrm{E}}$, as was done in [28]. However, we have limited ourselves here to estimating only the main Einstein temperature for each compound. In the inset of Fig. 7, we present the above function for the aluminides considered here, but dependent on $\log T$; we also mark the contributions originating from the electronic specific heat, $C_{\mathrm{e}}(T)$ using dashed lines. These temperatures are comparable to those found for the Fe-system studied previously [28].

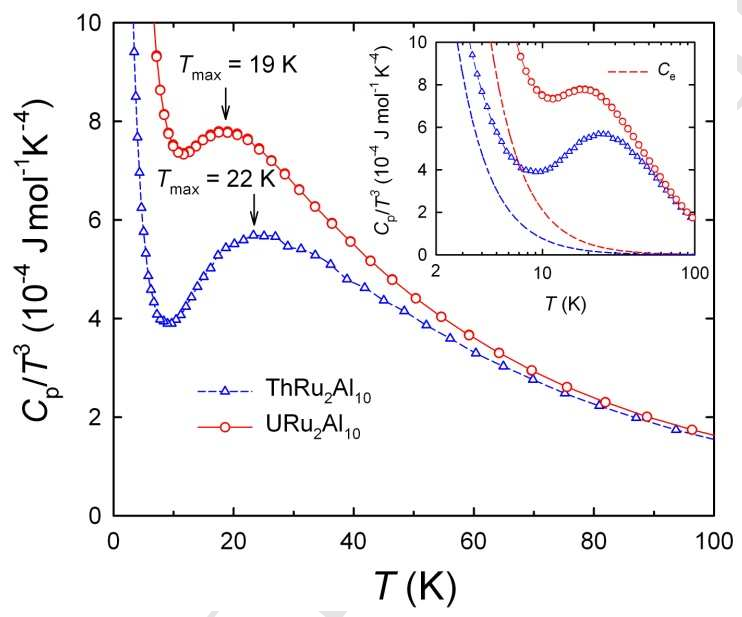

FIG. 7. The $C_{\mathrm{p}}(T) / T^{3}$ versus $T$ and $\log T$ (inset) dependencies for $(\mathrm{U}, \mathrm{Th}) \mathrm{Ru}_{2} \mathrm{Al}_{10}$. The dashed lines in the inset represent the electronic specific heat variation in the form of $C_{\mathrm{e}}(T) / T^{3}$ versus $\log T$.

For better recognition of the Schottky anomalies for both uranium systems containing Fe and $\mathrm{Ru}$, we plot in the upper part of Fig. 8 the CF level schemes obtained in our intermediate calculations for these two compounds; in the lower part of this figure, we present the corresponding theoretical Schottky anomalies, using the following expression:

$$
C_{\mathrm{Sch}}(T)=\frac{R}{T^{2}}\left[\frac{\sum_{i} E_{i}^{2} e^{-E_{i} / T}}{\sum_{i} e^{-E_{i} / T}}-\left(\frac{\sum_{i} E_{i} e^{-E_{i} / T}}{\sum_{i} e^{-E_{i} / T}}\right)^{2}\right],
$$

where the summation runs over the nine eigenvalues $E_{\mathrm{i}}(R$ is the universal gas constant).

The above $\mathrm{CF}$ schemes yield different temperature-dependent curves for the above aluminides below about $250 \mathrm{~K}$, and have almost the same temperature dependencies above this temperature. As can be seen, however, the maxima are different. The maximum for Fe-containing aluminide is broadened, while for the corresponding Ru-aluminide it is much sharper. In our previous paper [28], we found fairly good agreement between the theoretical and experimental data for $\mathrm{UFe}_{2} \mathrm{Al}_{10}$ (not shown here). It seems that we have a similar agreement for the data for $\mathrm{URu}_{2} \mathrm{Al}_{10}$ presented here, despite the experimental difficulties described earlier in this text regarding the estimation of an anomaly in multi-atomic compounds. In the same figure, we plot the magnetic entropy, which increases smoothly to a value slightly below $R \ln 7$ at $600 \mathrm{~K}$ for both systems. 


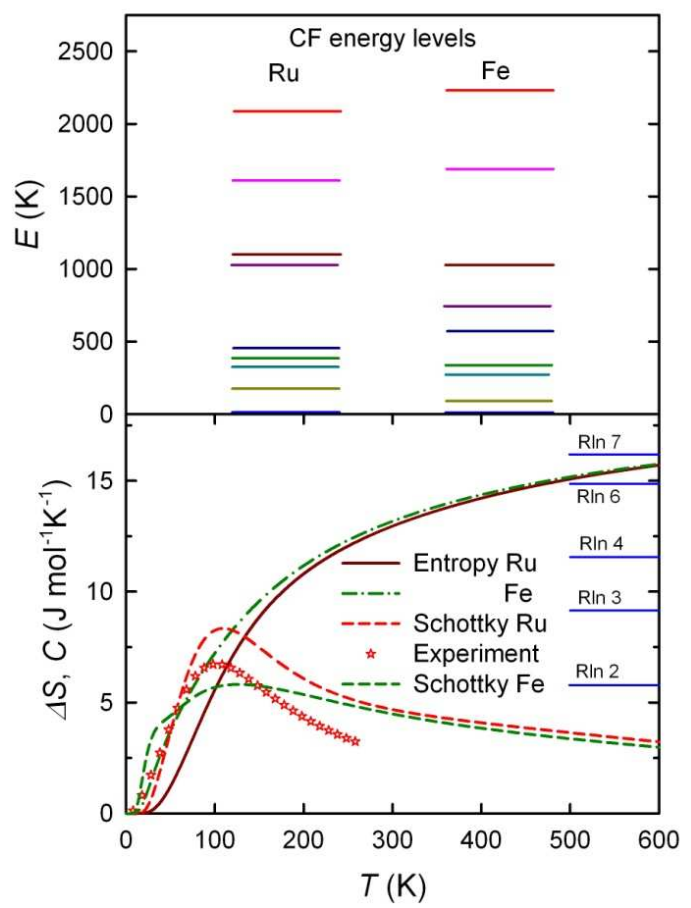

Fig. 8. Upper panel: Crystal field schemes for $\mathrm{URu}_{2} \mathrm{Al}_{10}$ (this work) and $\mathrm{UFe}_{2} \mathrm{Al}_{10}$ (Ref. [28]). Lower panel: Schottky anomaly (left hand scale) and magnetic entropy (right hand scale), both based on the given schemes of $\mathrm{CF}$ levels for $\mathrm{U}(\mathrm{Fe} ; \mathrm{Ru})_{2} \mathrm{Al}_{10}$.

\subsection{Transport properties}

\subsubsection{Electrical resistivity}

In Fig. 9, we plot the electrical resistivity $\rho_{\mathrm{i}}$ against temperature $T$, measured for a single crystal of $\mathrm{URu}_{2} \mathrm{Al}_{10}$, with the current $\boldsymbol{J}$ applied along the three main crystallographic axes $i=a, b$ and $c$. Note that all these curves have similar shapes, with rapidly increasing resistivity above $50 \mathrm{~K}$ and an inflection point, $T_{\mathrm{inf}}$, at around $80 \mathrm{~K}$, where the temperature derivative, $\mathrm{d} \rho_{i}(T) / \mathrm{d} T$, goes through a maximum (not shown here). As can be seen from this figure, $\rho_{i}(T)$ shows a strong tendency to saturation at temperatures above $150 \mathrm{~K}$, finally reaching the following values at RT: 280,290 and 250 $\mu \Omega \mathrm{cm}$ for the $a, c$ and $b$ axes, respectively. The corresponding residual resistivities $\rho_{0 i}$ are 14,12 and $5 \mu \Omega \mathrm{cm}$. It is also apparent from this figure that the $\rho_{a}(T)$ and $\rho_{c}(T)$ curves behave similarly and that the anisotropy relates mainly to the $b$ axis, for which $\rho_{b}(T)$ assumes lower values. Similar anisotropy was reported by Blanco et al. [66] in a system with hexagonal symmetry, namely $\mathrm{PrNi}_{5}$. These authors pointed out that a quadrupolar contribution plays an important role in the observed anisotropy. Thus, we can expect the same situation for the uranium systems with axial symmetry considered here; these possess an aspherical distribution of $5 f$ charges, which in turn also influences the magnetoresistivity through an anisotropic quadrupolar contribution.

Comparing these data for $\rho_{i}(T)$ to those previously reported for $\mathrm{UFe}_{2} \mathrm{Al}_{10}$ [32], we can see a very close similarity. In this figure, we also show the residual resistivity ratios (RRR), which are somewhat different for each axis of measurement and take values from 20 to 49 . The corresponding values for $\mathrm{UFe}_{2} \mathrm{Al}_{10}$ are between nine and 13 [32]. The inset of Fig. 9 shows the low-temperature $\rho_{i}$ vs. $T^{2}$ functions taken at zero magnetic field. These are practically straight lines (except for the lowest range of temperatures), indicating a Fermi liquid state. Table 6 gives the values of the residual resistivities $\rho_{0 i}$ and the corresponding coefficient $\mathrm{A}_{i}$ for $\mathrm{URu}_{2} \mathrm{Al}_{10}$ found for the three main axes, and a comparison of these values for $\mathrm{UFe}_{2} \mathrm{Al}_{10}$. Interestingly, the Kadowaki-Woods relation $\mathrm{A}_{\mathrm{a}} / \gamma(0)^{2} \approx(0.5-$ 1.3) $10^{-5}\left(\mu \Omega \mathrm{cm} \mathrm{K}^{2} \mathrm{~mol}^{2} \mathrm{~mJ}^{-2}\right)$ is followed here [67]. 


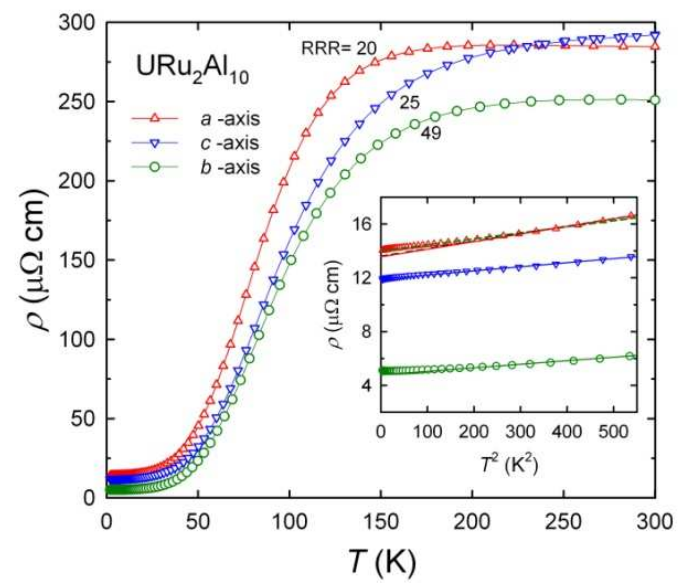

Fig. 9. Electrical resistivity of $\mathrm{URu}_{2} \mathrm{Al}_{10}$ as a function of temperature measured for the three main crystallographic axes. Inset: $\rho_{i}$ versus $T^{2}$. As is evidenced from the inset, the Fermi liquid state characterizes the $\mathrm{Ru}$-based aluminide. Here, however, the lowest resistivity values are found along the $b$ axis instead of the $c$-axis observed for $\mathrm{UFe}_{2} \mathrm{Al}_{10}[32]$.

The $\rho_{0 i}$ data taken at $9 \mathrm{~T}$ with the field applied perpendicular to the flowing current $\boldsymbol{J}$ are higher than the zero-field values, reaching respectively about 19, 14.5 and $8 \mu \Omega \mathrm{cm}$ (not shown here). These results indicate that the traverse magnetoresistance (TMR) is also positive, as for $\mathrm{UFe}_{2} \mathrm{Al}_{10}$ [32], and this is discussed below.

Table 6

Low-temperature electrical resistivity parameters.

\begin{tabular}{llllll}
\hline Compound & axis & $\begin{array}{l}\rho_{0}^{\exp } \\
(\mu \Omega \mathrm{cm})\end{array}$ & $\begin{array}{l}\rho_{\text {min }} \\
(\mu \Omega \mathrm{cm})\end{array}$ & $\begin{array}{l}\rho_{0}^{\text {calc }} \\
(\mu \Omega \mathrm{cm})\end{array}$ & $\begin{array}{l}\mathrm{A}_{i} \\
\left(\mu \Omega \mathrm{cm} \mathrm{K}^{-2}\right)\end{array}$ \\
\hline $\mathrm{UFe}_{2} \mathrm{Al}_{10}[32]$ & $a$ & 32.5 & 32.4 & 31.7 & 0.00495 \\
& $b$ & 28.7 & & 29.7 & 0.00331 \\
$\mathrm{URu}_{2} \mathrm{Al}_{10}$ & $c$ & 22.6 & & 22.3 & 0.00340 \\
& $a$ & 13.96 & & 13.6 & 0.00558 \\
& $b$ & 5.11 & 5.08 & 4.9 & 0.00221 \\
& $c$ & 11.90 & & 12.0 & 0.00281 \\
\hline
\end{tabular}

The observed shape of the $\rho_{i}(T)$ functions of $\mathrm{URu}_{2} \mathrm{Al}_{10}$, as for $\mathrm{UFe}_{2} \mathrm{Al}_{10}$ [32], is reminiscent of those measured for several intermetallic compounds containing isoelectronic ions to $\mathrm{U}^{4+}$, for example $\mathrm{Pr}^{3+}$. Other examples are cubic $\operatorname{PrIn}_{3}$ [68], hexagonal $\operatorname{PrCu}_{5}$, orthorhombic $\operatorname{PrCu}_{6}$ [69], and a number of other metallic $\mathrm{Pr}^{3+}$ systems showing the $\mathrm{CF}$ effect. It is worth mentioning that of the caged systems such as the $\operatorname{PrRu}_{4} \mathrm{As}_{12}$ skutterudite [70] and others, the $\rho(T)$ function has also been analyzed in terms of the CF model. None of these systems show magnetic ordering, down to the lowest temperature measured, since their ground state is a non-magnetic CF singlet. The orthorhombic uranium ternaries considered in this paper also have this ground state. Good quantitative agreement between theory and experiment was obtained for all these examples following the subtraction of a phonon component from their total resistivities using the data of the corresponding isostructural non-magnetic counterpart, namely $\mathrm{ThFe}_{2} \mathrm{Al}_{10}$ (see e.g. [28]). From the previous measurements, we assume that the dramatic jump in resisitivity at about $50 \mathrm{~K}$ is unrelated to any magnetic order, spin fluctuations, mixed valence state or coherent Kondo effect, which are often cited in the literature as explanations for similar shapes of the $\rho(T)$ behaviors of many intermetallic systems. Unfortunately, the phonon contribution is not taken into account in our data for $\mathrm{URu}_{2} \mathrm{Al}_{10}$ due to the lack of a suitably sized single crystal of $\mathrm{ThRu}_{2} \mathrm{Al}_{10}$ to carry out such measurements. However, the inclusion of the phonon contribution would cause the high-temperature slope $\mathrm{d} \rho / \mathrm{d} T$ to be negative. This, in turn, would indicate some Kondo-like participation in the electron scattering effect, but this can hardly be considered a Kondo effect in singlet CF ground state materials. Nevertheless, this problem was considered in [71] for the case of $\mathrm{PrSn}_{3}$ and its solid solutions with $\mathrm{LaSn}_{3}$. As pointed out by Kuramoto et al. [72], the CF singlet ground 
state can give way to Kondo singlets through exchange interaction with the conduction band (see also [28]).

The close similarity between the $\rho_{i}(T)$ (Fig. 9) and those presented earlier for $\mathrm{UFe}_{2} \mathrm{Al}_{10}$ (see Fig. 9 of [32]) and the results of theoretical calculations for the latter aluminide (see Fig. 12 of [28]) gives rise to the conclusion that for agreement with experiment, we have to include some Kondo-like interactions in both uranium ternaries considered here (apart from the $\mathrm{CF}$ and quadrupolar effects). For further discussion of this problem, see [28,32].

We also performed measurements of transverse magnetoresistivity (TMR) for $\mathrm{URu}_{2} \mathrm{Al}_{10}$, defined as $[\Delta \rho / \rho(0)]=100 \times[\rho(B)-\rho(0)] / \rho(0)(\%)$. These measurements were carried out over a wide range of temperatures (from 2 to $80 \mathrm{~K}$ ) and magnetic fields up to $9 \mathrm{~T}$, for the current $\boldsymbol{J}_{i}$ flowing along the three main crystallographic axes and arranged perpendicular to the applied magnetic field. The obtained results are analogous to those reported for $\mathrm{UFe}_{2} \mathrm{Al}_{10}$ (see Fig. 10 in [32]). In both cases, the field variation of TMR displays a typical metallic $A_{i}(T) B^{\mathrm{n}}$ dependence with increasing magnetic field strength, where the exponent $\mathrm{n}$, detected for the three main directions $i=a, b$ and $c$, lies in the ranges $1.86-1.23,1.79-1.50$ and 1.75-1.33, respectively, when the temperature changes from 2 to $80 \mathrm{~K}$. These results indicate that the curves show a more parabolic character at low temperatures, while at higher temperatures they evolve to almost linear behavior. For all these three cases, the $\Delta \rho / \rho_{\mathrm{i}}(0)_{\mathrm{i}}(\%)$ vs. $B$ function is positive (not shown here) and the coefficient $A_{\mathrm{i}}(T)$ decreases smoothly with increasing temperature for each case.
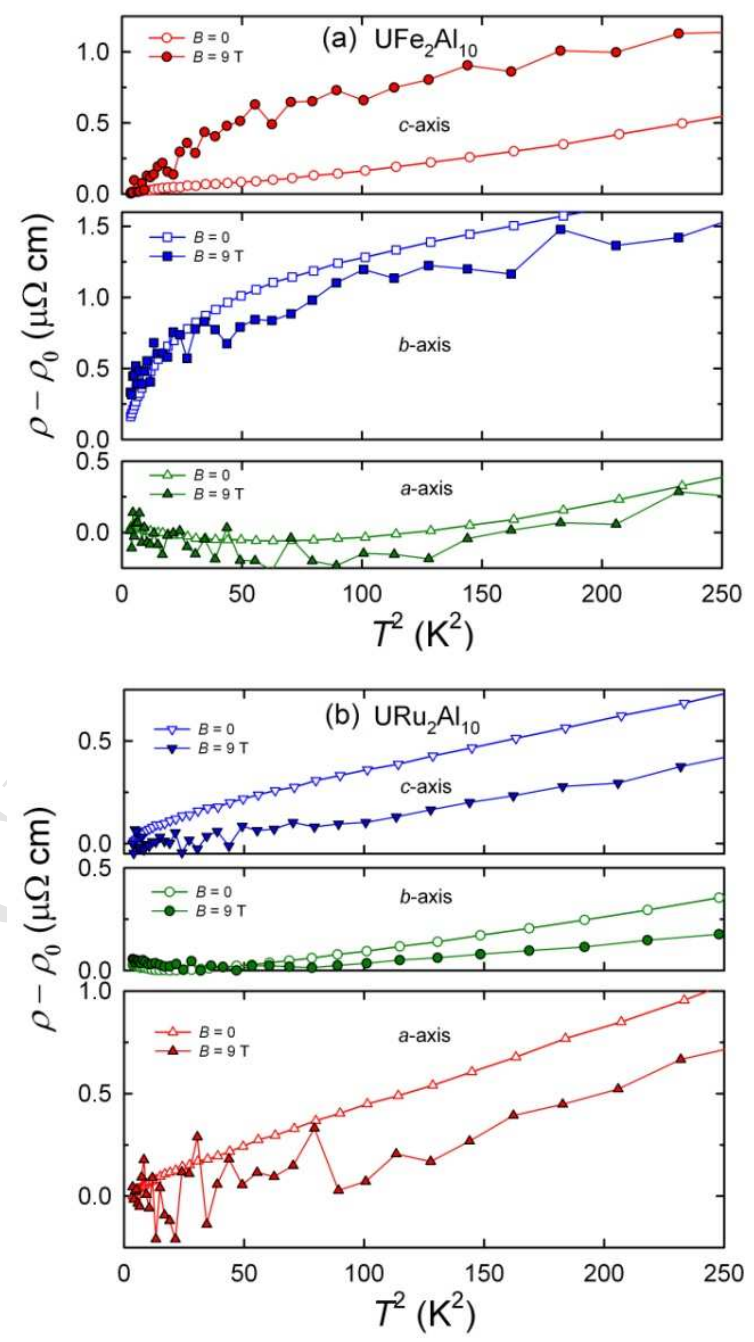

Fig. 10. $\rho-\rho_{0}$ vs. $T^{2}$ functions measured at 0 and $9 \mathrm{~T}$ for three main crystallographic axes for (a) $\mathrm{UFe}_{2} \mathrm{Al}_{10}$ and (b) $\mathrm{URu}_{2} \mathrm{Al}_{10}$. Note that external magnetic field disturbs a smooth behavior of the resistivity measured at zerofield, owing to the electron-rattling interaction (see the text). 
The main aim of this work focuses on a comparison of the resistivity behavior dependent on $T^{2}$ (after subtracting either the residual or minimum resistivity from the total resistivity (see Table 6)). These data were taken at zero and $9 \mathrm{~T}$ and are plotted in Figs. 10(a) and 10(b) for the Fe- and Rubased compounds, respectively. Thus, these figures present the results obtained for a temperature region of below about $16 \mathrm{~K}$. As expected based on our earlier results obtained for $\mathrm{UB}_{12}$ [11] and $\mathrm{ThFe}_{2} \mathrm{Al}_{10}$ [28], $\mathrm{UFe}_{2} \mathrm{Al}_{10}$ and $\mathrm{URu}_{2} \mathrm{Al}_{10}$ also reveal a new phenomenon under an applied field of $9 \mathrm{~T}$, for example. This phenomenon has already been described by the current authors, and is associated with a large scattering of the experimental resistivity points at low temperatures after applying an external magnetic field. This effect is strong in one crystallographic direction, and weak in both others. However, measurements taken at zero field give very smooth $\rho_{i}(0)$ vs $T^{2}$ curves, where the size of the experimental point represents the accuracy of the measurements. We would like to emphasize that the data in Fig. 10 unambiguously indicate that the applied field substantially influences the rattling of the central ion inside its oversized $\left[T_{4} \mathrm{Al}_{16}\right]$ cage, causing its chaotic motion. This effect is the result of an existing interaction between the rattling and the conduction band. This interaction, called "electronrattling (e-r)", has already been described for other caged-type compounds, such as the 1:2:20 intermetallics [7]. As is the case for $\mathrm{ThFe}_{2} \mathrm{Al}_{10}$ [28], this phenomenon is strongly anisotropic and vanishes rapidly with increasing temperature. The largest scattering effect is clearly seen for $\mathrm{URu}_{2} \mathrm{Al}_{10}$ along the $a$-axis, which is also the axis of the largest $c-f$ hybridization, and is perpendicular to the easy $c$-axis [21]. Further details of this type of interaction and its influence on the physical properties of the caged-type compounds will, we hope, be given in the future. Various types of modern investigation will allow for a much better understanding of the localized character of the $5 f$ electrons in a metallic surrounding and a recognition of the mechanism of their dual nature. Until now, except for $\mathrm{UPd}_{3}$ which was considered for many years to be a canonical system of this behavior, only a few examples have been reported, such as UPdSn, UNiSn and several other complex uranium intermetallics, for which neutron inelastic measurements confirmed the presence of the crystal field excitations [73]. However, in contrast to the 1:2:10 ternaries considered here, all the systems mentioned above exhibit either magnetic, quadrupolar or structural transitions at low temperatures, which makes the interpretation of any obtained data much more complicated. For example, $\mathrm{UPd}_{3}$ exhibits as many as four different quadrupolar transitions at low temperatures [74].

Fig. 11 shows the thermal behavior of TMR (open small symbols) taken at $9 \mathrm{~T}$ for singlecrystalline samples of $\mathrm{URu}_{2} \mathrm{Al}_{10}$.

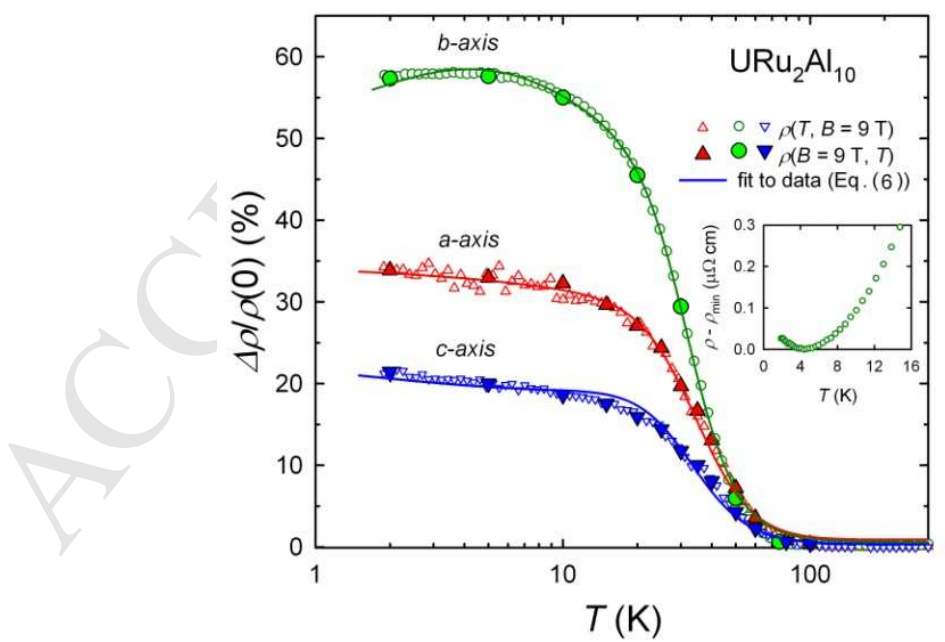

Fig. 11. Transverse magnetoresistivity of $\mathrm{URu}_{2} \mathrm{Al}_{10}$ as a function of temperature along three main crystallographic axes $i$. Solid lines denote fittings to Eq. (6) (in the range $2-100 \mathrm{~K}$ ) with parameters a $=0.317$, 0.546 , and $\left.0.865\left[\mathrm{~T}^{2}(\mu \Omega \mathrm{cm})^{-1}\right)\right], \mathrm{b}=-0.025,-0.017$, and $\left.-0.078\left[\mathrm{~T}^{2}(\mu \Omega \mathrm{cm})^{-1}\right)\right]$ for axes $a, b$, and $c$, respectively. In the inset see a minimum in $\rho_{b}(T)$ at low temperatures.

We also plot on these smoothed curves the results at $9 \mathrm{~T}$ (closed large symbols) selected from the $\Delta \rho / \rho(0)_{i}$ vs. $B$ dependencies (not shown here). As can be seen, both measurements are in good agreement. These curves first decrease smoothly and then rapidly, almost to zero, with increasing 
temperature, as for $\mathrm{UB}_{12}$ [11]. Note that the curve for $i=a$ shows a distinct scattering of the experimental points, in agreement with the data shown in Fig. 10(b). The flat maximum in TMR observed for the $b$-axis at about $5 \mathrm{~K}$ arises due to a diffuse minimum being reached at this temperature by $\rho_{b}(T)$, as shown in the inset of Fig. 11.

As Fig. 11 shows, the $\Delta \rho / \rho(0)_{i}$ versus $T$ dependencies (where $i=a, b$ and $c$ ) taken at $9 \mathrm{~T}$ for $\mathrm{URu}_{2} \mathrm{Al}_{10}$ can be fitted fairly well by Eq. (6) (solid lines):

$$
\frac{\Delta \rho_{i}}{\rho_{i}(0)}=\frac{B^{2}}{\mathrm{a}_{i}\left[\rho_{i}(0, T)\right]+\mathrm{b}_{i} B^{2}},
$$

where $\mathrm{a}_{i}$ and $\mathrm{b}_{i}$ are the field- and temperature-independent parameters, which depend exclusively on conduction electron properties, and $\rho_{\mathrm{i}}(0, T)$ is the total resistivity at zero field. The above formula describes so-called normal magnetoresistance, i.e. the influence of the magnetic field on the conduction electron trajectories (the so-called Lorentz effect) [75]. This mechanism always gives rise to positive TMR, the magnitude of which increases for a reduction in temperature for a fixed finite field. This effect is due to the simultaneous decrease in electron-phonon scattering, which always falls when the temperature is decreased. The derived parameters of Eq. (6) are given in the caption to Fig. 11. The anisotropy in the temperature variation of TMR observed for our aluminides seems to be caused by the $c-f$ hybridization, which is also strongly anisotropic. It was deduced that the $c-f$ hybridization for this material is large in the $a c$-plane but very small along the $b$-axis. It is puzzling that one consequence of the anisotropy mentioned above is the large difference between the Fe- and $\mathrm{Ru}$ - counterparts in the value of TMR, e.g. in the direction of the $b$-axis. For the latter, we find the highest value of about $60 \%$, while for the former the lowest value is about $4 \%$ (see Fig. 11 in [32]). Another difference is the lack of a maximum in $\Delta \rho / \rho(0)_{i}$ vs. $T$, observed only for $\mathrm{UFe}_{2} \mathrm{Al}_{10}$ along the $c$ axis of the flowing current. An explanation for these differences requires deeper study and the consideration of data for $\mathrm{UOs}_{2} \mathrm{Al}_{10}$, which are now in preparation.

\subsubsection{Thermoelectric power}

The thermoelectric power (TEP), $S_{i}(T)$, of $\mathrm{URu}_{2} \mathrm{Al}_{10}$ was also measured along the three main crystallographic axes, and the results are displayed in Fig. 12.

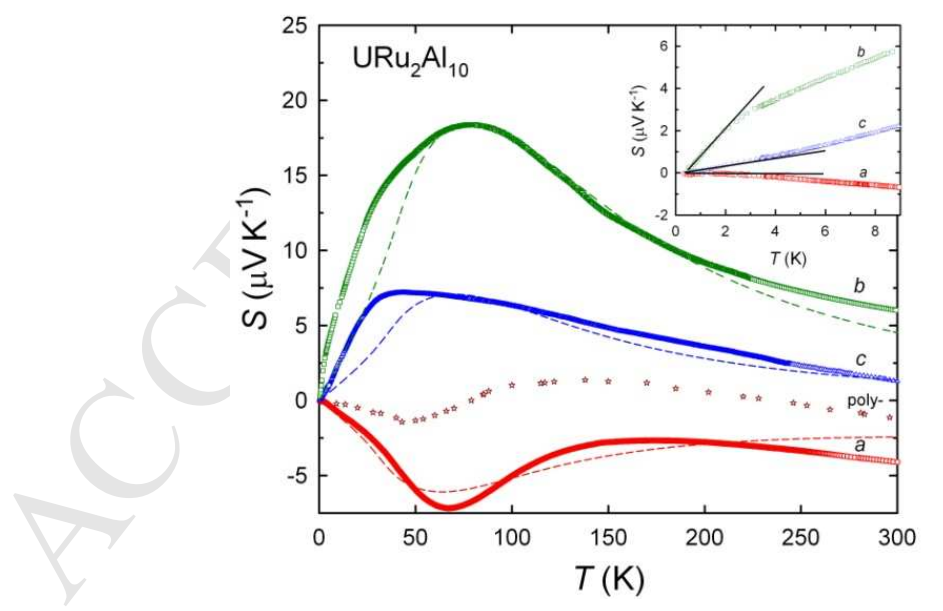

Fig. 12. Thermoelectric power of $\mathrm{URu}_{2} \mathrm{Al}_{10}$ as function of temperature measured along three crystallographic $i$ directions. Dashed lines denote fits of Eq. (7) to $S_{i}(T)$ data with parameters given in Table 7. Inset: lowtemperature thermoelectric power $S_{i}(T)$. Solid lines represent the slopes, $\mathrm{d} S_{i} / \mathrm{d} T$, drawn in the temperature range from 0.4 to $3.5 \mathrm{~K}$. The numerical values of the slopes are also given in Table 7.

As can be seen, all these curves are strongly dependent on temperature, and show distinct broad maxima at 65,75 and $35 \mathrm{~K}$ for a temperature gradient $\Delta T$ directed along the $a$-, $b$ - and $c$ - axes, respectively. Across the temperature region measured (0.4 to $300 \mathrm{~K}), S_{b}(T)$ and $S_{c}(T)$ are positive, while $S_{a}(T)$ is negative. After reaching a pronounced positive maximum with a value of $18.5 \mu \mathrm{V} \mathrm{K}^{-1}$, 
$S_{b}(T)$ starts to smoothly decrease further, reaching about $6.5 \mu \mathrm{V} \mathrm{K} \mathrm{K}^{-1}$ at RT. We previously found a very similar temperature variation in TEP along the $b$-axis in the case of $\mathrm{UFe}_{2} \mathrm{Al}_{10}$ [32]. The similarity in the TEP of both ternaries also exists in $S_{a}(T)$, since their temperature variations are negative and have broad negative maxima at almost the same temperature of about $65 \mathrm{~K}$; however, the values reached at this temperature are different, namely -12 and $-7.5 \mu \mathrm{V} \mathrm{K} \mathrm{K}^{-1}$ for the $\mathrm{Fe}$ - and Ru-based aluminides, respectively. Moreover, both curves display very diffuse negative minima at about 110 and $150 \mathrm{~K}$, finally reaching values of -18 and $-4 \mu \mathrm{V} \mathrm{K}^{-1}$ at $\mathrm{RT}$. However, the largest difference is shown in the behavior of $S_{c}(T)$. This function is negative for $\mathrm{UFe}_{2} \mathrm{Al}_{10}$ across the whole temperature region measured, first showing a deep maximum at about $65 \mathrm{~K}$ with a large value of $-28 \mu \mathrm{V} \mathrm{K}^{-1}$, and then a diffuse minimum at about $200 \mathrm{~K}$, and finally reaching a value of $-18 \mu \mathrm{V} \mathrm{K}^{-1}$ at $\mathrm{RT}$, which coincides with that of $S_{a}(T)$. The behavior of the corresponding curve for $\mathrm{URu}_{2} \mathrm{Al}_{10}$ is the opposite; it is entirely positive, reaching a maximum at about $45 \mathrm{~K}$ with a value of $7.5 \mu \mathrm{V} \mathrm{K}^{-1}$, and falls almost linearly to a value of $1.8 \mu \mathrm{V} \mathrm{K}^{-1}$ at RT. Fig. 12 shows the temperature variation of TEP for the polycrystalline sample of $\mathrm{URu}_{2} \mathrm{Al}_{10}$, the data for which we reported several years ago [34]. This curve reflects the shape of $S_{\mathrm{a}}(T)$, with a very shallow negative maximum at about $50 \mathrm{~K}$ and a positive maximum at about $150 \mathrm{~K}$.

Above this temperature, $S_{\text {poly }}(T)$ reaches a magnitude close to zero at RT. This comparison clearly reveals that strong anisotropy in TEP does exist in the $\mathrm{U}(\mathrm{Fe} ; \mathrm{Ru})_{2} \mathrm{Al}_{10}$ ternaries.

We also plot all three low temperature $S_{\mathrm{i}}(T)$ curves in the inset of Fig. 12, and draw their slopes, $\mathrm{d} S_{i} / \mathrm{d} T$, the values of which are given in Table 7 . As this inset shows, an approximate zero value is achieved by this function at about $T \approx 0.4 \mathrm{~K}$. Hence, we can infer the probability of a superconducting state occurring in $\mathrm{URu}_{2} \mathrm{Al}_{10}$ just below this temperature. In Fig. 12, we also mark (with dashed curves) the calculated total thermoelectric power $S_{i}(T)$ by applying Eq. (7), as proposed by Fulde and Peschel [76].

$$
S(T)=S_{\mathrm{d}}(T)+S_{\mathrm{CF}}(T)=\mathrm{A} T+\text { constant } \times F(T / \delta)
$$

where $S_{\mathrm{d}}$ and $S_{\mathrm{CF}}$ are the two main contributions to the total TEP, i.e. the diffuse and CF effect contributions, respectively. $A=\xi \pi^{2} k_{B}^{2} / e E_{F}$, where $\xi$ is the thermoelectric parameter [77]. $F(T / \delta)$ is a universal function with a maximum at $T_{\max } \approx 0.3 \delta$, where $\delta$ is the energy distance between the ground and first (or closed group) excited CF levels. The fitting parameters are listed in Table 7. As can be seen from Fig. 12, the fitting curves (dashed lines) closely reproduce all the observed extrema in the $S_{\mathrm{i}}(T)$ curves. Nevertheless, this fitting should be treated with some caution. In Equation (7) above, the possible presence of the phonon drag $\left(S_{\mathrm{g}}\right)$ has been omitted, and the derived energy splitting between the ground and the first excited CF level(s) should be treated as an approximate value. More detailed studies including an external magnetic field may give a better view of the TEP in these aluminides.

\section{Table 7}

Fitting parameters of Eq. (7) to thermoelectric power $S_{\mathrm{i}}(T)$ data and the slopes $\mathrm{d} S_{i}(T) / \mathrm{d} T$, calculated in the temperature range $\mathrm{d} T=3.5-0.4 \mathrm{~K}$.

\begin{tabular}{lllll}
\hline Axis & $A_{i}\left(\mu \mathrm{V} \mathrm{K}^{-2}\right)$ & Const. $\left(\mu \mathrm{V} \mathrm{K}^{-1}\right)$ & $\delta(\mathrm{K})$ & $\mathrm{d} S_{i}(T) / \mathrm{d} T\left(\mu \mathrm{V} \mathrm{K}^{-2}\right)$ \\
\hline$a$ & -0.00524 & -6.62 & 242 & -0.05 \\
$b$ & -0.00014 & 21.28 & 311 & 1.29 \\
$c$ & -0.00014 & 8.13 & 276 & 0.22 \\
\hline
\end{tabular}

\section{Conclusions}

We examine another uranium ternary compound from the 1:2:10 series with a cage-type structure, namely $\mathrm{URu}_{2} \mathrm{Al}_{10}$. This can be regarded as being simultaneously both a metallic system and a Van Vleck paramagnet, without a tendency to magnetic ordering down to the lowest temperature studied of $0.4 \mathrm{~K}$. This behavior was inferred in the same way as that previously estimated for $\mathrm{UFe}_{2} \mathrm{Al}_{10}$, from bulk measurements performed on single-crystalline samples of $\mathrm{URu}_{2} \mathrm{Al}_{10}$. It was found that $5 f$ electrons of uranium form two different subsets: one is located around $E_{\mathrm{F}}$ in the conduction band, and the other has a binding energy below $E_{\mathrm{F}}$. The former state was closely reproduced by band 
structure calculations reported earlier [35], while the latter, with a $5 f^{2}$ configuration, represents a fully localized character. This state was therefore confirmed using CF potential calculations, in which the complex intermediate coupling method was used; this is indispensable in the case of CF probing of the $5 f$ electron system. The obtained CF level splitting scheme enables us to reproduce the strong anisotropy in the temperature variation of the magnetic susceptibility. We also find the Schottky-type data is reproduced well in this scheme; this was extracted for $\mathrm{URu}_{2} \mathrm{Al}_{10}$ in a similar way as for $\mathrm{UFe}_{2} \mathrm{Al}_{10}$ [32]. The extraordinarily high values of six-rank CF parameters estimated for both the compounds and the divergence of these with $\mathrm{k} \leq 4$ with respect to the cerium analogue can be explained as a consequence of the dual nature of the $f$ electrons. In addition to the magnetic and specific heat results, we also carried out electron transport measurements. The evolution of the temperature dependence of the resistivity clearly suggests the dominance of the CF effect in its shape, as shown for its Fe-counterpart [28]. This effect is also predominant in the thermoelectric power data, leading to the occurrence of the large maxima in $S_{\mathrm{i}}(T)(i=a, b$ and $c)$ in a similar manner to that described previously for $\mathrm{UFe}_{2} \mathrm{Al}_{10}$ [32]. In addition, we attain further confirmation of the dual character of the $5 f$ electrons in $\mathrm{URu}_{2} \mathrm{Al}_{10}$ by analyzing the transverse magnetoresisistivity. This reveals that the rattling of the $\mathrm{U}^{4+}$ ion in the $\left[\mathrm{Ru}_{4} \mathrm{Al}_{16}\right]$ cage is strongly disturbed by the application of a magnetic field, which gives rise to anisotropic behavior in terms of the substantial scattering of the experimental resistivity points detected only at low temperatures. This effect was observed for the first time in another caged uranium compound, $\mathrm{UB}_{12}$ [11]. This can arise from the interaction between the rattling of the central atom and the conduction band, and this has also been described for other cagedtype compounds such as ternaries with 1:2:20 stoichiometry [7]. We believe that this new effect merits further detailed study. Moreover, we regard the $\mathrm{UT}_{2} \mathrm{Al}_{10}$ ternaries as excellent materials to be examined by more advanced methods, in view of their specific electronic structure (dualism) and the rattling of the $\mathrm{U}^{4+}$ ion in its oversized cage.

\section{Acknowledgments}

The authors are grateful to the IT Centre at the Institute of Low Temperature and Structure Research of the Polish Academy of Sciences in Wrocław for the use of supercomputers and technical support.

\section{References}

[1] H. Kusunose, K. Miyake, Two-channel Kondo model as a fixed point of local electron-phonon coupling system, J. Phys. Soc. Jpn. 65 (1996) 3032.

[2] K. Hattori, Y. Hirayama, K. Miyake, Local heavy quasiparticle in four-level Kondo model, J. Phys. Soc. Jpn. 74 (2005) 3306.

[3] Z. Hiroi, Y. Yamaura, K. Hattori, Rattling good superconductor: $\beta$-pyrochlore oxides $\mathrm{AOs}_{2} \mathrm{O}_{6}$, J. Phys. Soc. Jpn. 81 (2012) 011012.

[4] V.M.T. Thiede, T. Ebel, W. Jeitschko, J. Mater. Chem. 8 (1998) 125.

[5] S. Niemann, W. Jeitschko, Ternary aluminides $A T_{2} \mathrm{Al}_{20}(A=$ rare earth elements and uranium; $T=$ $\mathrm{Ti}, \mathrm{Nb}, \mathrm{Ta}, \mathrm{Mo}$, and W) with $\mathrm{CeCr}_{2} \mathrm{Al}_{20}$-type structure, J. Solid State Chem. 114 (1995) 337;

S. Niemann, W. Jeitschko, The crystal structure of $\mathrm{YbFe}_{2} \mathrm{Al}_{10}$, a combined substitution and stacking variant of the $\mathrm{ThMn}_{12}$ and $\mathrm{CeMn}_{4} \mathrm{Al}_{8}$ type structures, Z. Kristallogr. 210 (1995) 338.

[6] Z. Hiroi, A. Onosaka, Y. Okamoto, J. Yamaura, H. Harima, Rattling and superconducting properties of the cage compound $\mathrm{Ga}_{x} \mathrm{~V}_{2} \mathrm{Al}_{20}$, J. Phys. Soc. Jpn. 81 (2012) 124707.

[7] A. Onosaka, Y. Okamoto, J. Yamaura, Z. Hiroi, Superconductivity in the Einstein solid $A_{x} V_{2} A l_{20}$ $(\mathrm{A}=\mathrm{Al}$ and Ga), J. Phys. Soc. Jpn. 81 (2012) 023703.

[8] M.J. Winiarski, B. Wiendlocha, M. Sternik, P. Wiśniewski, J.R. O’Brien, D. Kaczorowski, T. Klimczuk, Rattling-enhanced superconductivity in $M \mathrm{~V}_{2} \mathrm{Al}_{20}(M=\mathrm{Sc}, \mathrm{Lu}, \mathrm{Y})$ intermetallic cage compounds, Phys. Rev. B 93 (2016) 134507.

[9] J. Teyssier, A.B. Kuzmenko, D. van der Merel, F. Marsiglio, A.B. Liashchenko, N. Shitsevalova, V. Filippov, Optical study of electronic structure and electron-phonon coupling in $\mathrm{ZrB}_{12}$, Phys. Rev. B 75 (2007) 134503.

[10] N.E. Sluchanko, A.N. Azarevich, M.A. Anisimov, A.V. Bogach, S.Yu. Gavrilkin, M.I. Gilmanov, V.V. Glushkov, S.V. Demishev, A.L. Khoroshilov, A.V. Dukhnenko, K.V. Mitsen, N.Yu. Shitsevalova, V.B. Filippov, V.V. Voronov, K. Flachbart, Suppression of 
superconductivity in $\mathrm{Lu}_{\mathrm{x}} \mathrm{Zr}_{1-\mathrm{x}} \mathrm{B}_{12}$ : Evidence of static magnetic moments induced by nonmagnetic impurities, Phys. Rev. B 93 (2016) 085130.

[11] R. Troć, R. Wawryk, A. Pikul, N. Shitsevalova, Physical properties of cage-like compound $\mathrm{UB}_{12}$, Phil. Mag. B 95 (2015) 2343.

[12] P. Swatek, D. Kaczorowski, Magnetic and electrical properties of $\mathrm{UCr}_{2} \mathrm{Al}_{20}$ single crystals, J. Solid State Chem. 191 (2012) 191.

[13] C.H. Wang, J.M. Lawrence, E.D. Bauer, K. Kothapalli, J.S. Gardner, F. Ronning, K. Gofryk, J.D. Thompson, H. Nakotte, F. Trouw, Unusual signatures of the ferromagnetic transition in the heavy fermion compound $\mathrm{UMn}_{2} \mathrm{Al}_{20}$, Phys. Rev. B 82 (2010) 094406.

[14] P. Wiśniewski, P. Swatek, A. Gukasov, D. Kaczorowski, Ferromagnetism in $\mathrm{UMn}_{2} \mathrm{Al}_{20}$ studied with polarized neutron diffraction and bulk magnetic measurements, Phys. Rev. B 86 (2012) 054438.

[15] A. Uziel, A.I. Bram, A. Venkert, A.F. Kiv, D. Fuks, L. Meshi, Abrupt symmetry decrease in the $\mathrm{ThT}_{2} \mathrm{Al}_{20}$ alloys $(\mathrm{T}=3 d$ transition metal), J. Alloys Compd. 648 (2015) 353.

[16] M.J. Winiarski, J.-C. Griveau, E. Colineau, K. Wochowski, P. Wiśniewski, D. Kaczorowski, R. Caciuffo, T. Klimczuk, Synthesis and properties of $A_{\mathrm{x}} \mathrm{V}_{2} \mathrm{Al}_{20}(A=\mathrm{Th}, \mathrm{U}, \mathrm{Np}$., Pu $)$ ternary actinide aluminides, J. Alloys Compd. 696 (2017) 1113.

[17] P. Swatek, D. Kaczorowski, Magnetic behavior in $\mathrm{UFe}_{2} \mathrm{Zn}_{20}$ and $\mathrm{URu}_{2} \mathrm{Zn}_{20}$ single crystals, J. Phys.: Condens. Matter 23 (2011) 466001.

[18] E.D. Bauer, C. Wang, V.R. Fanelli, J.M. Lawrence, E.A. Goremychkin, N.R. de Souza, F. Ronning, J.D. Thompson, A.V. Silhanek, V. Vildosola, A.M. Lobos, A.A. Aligia, S. Bobev, J.L. Sarrao, Simplifying strong electronic correlations in uranium: Localized uranium heavyfermion $\mathrm{UM}_{2} \mathrm{Zn}_{20}(M=\mathrm{Co}, \mathrm{Rh})$ compounds, Phys. Rev. B 78 (2008) 115120.

[19] P. Swatek, M. Daszkiewicz, D. Kaczorowski, Paramagnetic heavy-fermion ground state in singlecrystalline $\mathrm{UIr}_{2} \mathrm{Zn}_{20}$, Phys. Rev. B 85 (2012) 094426.

[20] H. Tanida, Y. Nonaka, D. Tanaka, M. Sera, Y. Kawamura, Y. Uwatoko, T. Nishioka, M. Matsumura, Magnetic anisotropy of Kondo semiconductor $\mathrm{Ce}_{2} \mathrm{Al}_{10}(T=\mathrm{Ru}, \mathrm{Os})$ in the ordered state, Phys. Rev. B 85 (2012) 205208.

[21] M. Sera, D. Tanaka, H. Tanida, C. Moriyoshi, M. Ogawa, Y. Kuroiwa, T. Nishoka, M. Matsumura, J. Kim, N. Tsun, M. Takata, Crystal structure and anisotropic $c-f$ hybridization in $\mathrm{CeT}_{2} \mathrm{Al}_{10}(\mathrm{~T}=\mathrm{Ru}, \mathrm{Fe})$, J. Phys. Soc. Jpn. 82 (2013) 024603.

[22] K. Hanzawa, Crystalline electric field effects in $\mathrm{Ce}_{2} \mathrm{Al}_{10}(T=\mathrm{Ru}, \mathrm{Os})$, J. Phys. Soc. Jpn. 80 (2011) 023707;

D.T Adroja, A.D. Hillier, Y. Muro, T. Takabatake, A.M. Strydom, A. Bhattacharyya, A. DaoudAladin, J.W. Taylor, Muon-spin-relaxation and inelastic neutron scattering investigations of the cage-type Kondo semimetals: $\mathrm{CeT}_{2} \mathrm{Al}_{10}$ (T = Fe, Ru and Os), Phys. Scr. 88 (2013) 068505.

[23] F. Strigari, T. Willers, Y. Muro, K. Yutani, T. Takabatake, Z. Hu, Y.-Y. Chin, S. Agrestini, H.J. Lin, C.T. Chen, A. Tanaka, M.W. Haverkort, L.H. Tjeng, A. Severing, Crystal-field ground state of the orthorhombic Kondo insulator $\mathrm{CeRu}_{2} \mathrm{Al}_{10}$, Phys. Rev. B 86 (2012) 081105(R).

[24] F. Strigari, T. Willers, Y. Muro, K. Yutani, T. Takabatake, Z. Hu, S. Agrestini, C.-Y. Kuo, Y.-Y. Chin, H.-J. Lin, T.W. Pi, C.T. Chen, E. Weschke, E. Schierle, A. Tanaka, M.W. Haverkort, L.H. Tjeng, A. Severing, Crystal field ground state of the orthorhombic Kondo semiconductors $\mathrm{CeOs}_{2} \mathrm{Al}_{10}$ and $\mathrm{CeFe}_{2} \mathrm{Al}_{10}$, Phys. Rev. B 87 (2013) 125119.

[25] S. Kimura, T. Iizuka, H. Miyazaki, T. Haijri, M. Matsunami, T. Mori, A. Irizawa, Y. Muro, J. Kajno, T. Takabatake, Optical study of charge instability in $\mathrm{CeRu}_{2} \mathrm{Al}_{10}$ in comparison with $\mathrm{CeOs}_{2} \mathrm{Al}_{10}$ and $\mathrm{CeFe}_{2} \mathrm{Al}_{10}$, Phys. Rev. B 84 (2011) 165125.

[26] Y. Muro, K. Motoya, Y. Saiga, T. Takabatake, Formation of a hybridization gap in a cage-like compound $\mathrm{CeFe}_{2} \mathrm{Al}_{10}$, J. Phys. Soc. Jpn. 78 (2009) 083707.

[27] A.M. Strydom, P. Peratheepan, Magnetism and electronic correlations in the iron aluminides $R \mathrm{Fe}_{2} \mathrm{Al}_{10}(R=\mathrm{Y}, \mathrm{Yb})$, Phys. Status Solidi RRL 12 (2010) 356.

[28] R. Troć, R. Wawryk, Z. Gajek, M. Pasturel, M. Samsel-Czekała, Comparative studies of the cage systems $\mathrm{ThFe}_{2} \mathrm{Al}_{10}$ and $\mathrm{UFe}_{2} \mathrm{Al}_{10}$, J. Alloys Compd. 727 (2017) 1302.

[29] P. Khuntia, A. Strydom, F. Steglich, M. Baenitz, Quenching of spin fluctuations in the $3 \mathrm{~d}$ and $4 \mathrm{f}$ aluminides $\mathrm{YFe}_{2} \mathrm{Al}_{10}$ and $\mathrm{YbFe}_{2} \mathrm{Al}_{10}$ : a comparative ${ }^{27} \mathrm{Al}$ NMR and specific heat study, Phys. Status Solidi B 250 (2013) 525; 
P. Khuntia, A.M. Strydom, L.S. Wu, M.C. Aronson, F. Steglich, M. Baenitz, Field-tuned critical fluctuations in $\mathrm{YFe}_{2} \mathrm{Al}_{10}$ : Evidence from magnetization, ${ }^{27} \mathrm{Al} \mathrm{NMR}$, and $\mathrm{NQR}$ investigations, Phys. Rev. B 86 (2012) 220401(R).

[30] K. Park, L.S. Wu, Y. Janssen, M.S. Kim, C. Marques, M.C. Aronson, Field-tuned Fermi liquid in quantum critical $\mathrm{YFe}_{2} \mathrm{Al}_{10}$, Phys. Rev. B 84 (2011) 094425.

[31] T. Sugai, Y. Haga, T.D. Matsuda, E. Yamamoto, N. Tateiwa, F. Honda, R. Settai, Y. Ōnuki, Single crystal growth and physical properties of ternary uranium compounds $\mathrm{U}_{2} \mathrm{Al}_{10}(M=\mathrm{Fe}$, Ru and Os), J. Phys.: Conf. Ser. 273 (2011) 012122.

[32] R. Troć, M. Samsel-Czekała, E. Talik, R. Wawryk, Z. Gajek, M. Pasturel, Electronic, magnetic, transport, and thermal properties of single-crystalline $\mathrm{UFe}_{2} \mathrm{Al}_{10}$, Phys. Rev. B 92 (2015) 104427.

[33] K.O. Kvashnina, H.C. Walker, N. Magnani, G.H. Lander, R. Caciuffo, Resonant x-ray spectroscopy of uranium intermetallics at the $M_{4,5}$ edges of uranium, Phys. Rev. B 95 (2017) 245103.

[34] R. Troć, M. Pasturel, O. Tougait, M. Potel, H. Noël, Crystal structure and physical properties of a new intermetallic compound $\mathrm{URu}_{2} \mathrm{Al}_{10}$, Intermetallics 19 (2011) 913.

[35] M. Samsel-Czekała, E. Talik, M. Pasturel, R. Troć, Electronic structure of cage-type ternaries $A \mathrm{Ru}_{2} \mathrm{Al}_{10}$ - theory and XPS experiment $(A=\mathrm{Ce}$ and U), J. Alloys Compd. 554 (2013) 438.

[36] G. Zwicknagl, A.N. Yaresko, P. Fulde, Microscopic description of origin of heavy quasiparticles in $\mathrm{UPt}_{3}$, Phys. Rev. B 65 (2002) 081103(R).

[37] G.M. Sheldrick, SADABS, Bruker AXS Inc. Madison, Wisconsin, USA, 2001.

[38] A. Altomare, M.C. Burla, M. Camalli, G.I. Cascarano, C. Giacowazzo, A. Guagliardi, A.G.G. Moliterni, G. Polidori, R. Spagna, SIR97: a new tool for crystal structure determination and refinement, J. Appl. Cryst. 32 (1999) 115.

[39] G.M. Sheldrick, A short history of SHELX, Acta Cryst. A 64 (2008) 112.

[40] E. Parthé, E.K. Cenzual, R. Gladyshevskii, Standardization of crystal-structure data as an aid to the classification of crystal-structure types, J. Alloys Compd. 197 (1993) 291.

[41] R. Wawryk, Z. Henkie, Low-temperature resistivity and thermoelectric power controlled by defects in the USb anitferromagnet, Phil. Mag. B 81 (2001) 223.

[42] E. Teatum, K. Gschneidner, J. Waber, Compilation of Calculated Data Useful in Predicting Metallurgical Behavior of The Elements in Binary Alloy Systems, LA-2345, Los Alamos Scientific Laboratory, 1960.

[43] H. Noël, A.P. Gonçalves, J.C. Waerenborgh, Characterization of the ternary uranium-iron aluminide $\mathrm{UFe}_{2} \mathrm{Al}_{10}$, Intermetallics 12 (2004) 189.

[44] H. Tanida, D. Tanaka, M. Sera, Ch. Moriyoshi, Y. Kuroiwa, T. Takesaka, T. Nishioka, H. Kato, M. Matsumura, Anisotropic transport properties of $\mathrm{CeRu}_{2} \mathrm{Al}_{10}$, J. Phys. Soc. Jpn. 79 (2010) 063709.

[45] K. Koepernik H. Eschrig, Full-potential nonorthogonal local-orbital minimum-basis bandstructure scheme, Phys. Rev. B 59 (1999) 1743 (FPLO9.00-34), www.FPLO.de;

H. Eschrig, M. Richter, I. Opahle, Relativistic solid state calculations, in: P. Schwerdtfeger (Ed.), Relativistic Electronic Structure Theory, Part 2. Applications (Theoretical and Computational Chemistry), vol. 14, Elsevier, Amsterdam, 2004, p 723.

[46] J.P. Perdew, Y. Wang, Accurate and simple analytic representation of the electron-gas correlation energy, Phys. Rev. B 45 (1992) 13244.

[47] T.D. Matsuda, Y. Haga, E. Yamamoto, S. Ikeda, H. Shishido, R. Settai, H. Harima, Y. Ōnuki, Single crystal growth and Fermi surface property in ThRhIn ${ }_{5}$, J. Phys. Soc. Jpn. 76 (2007) 064712.

[48] H. Tanida, M. Nakamura, M. Sera, A. Kondo, K. Kindo, T. Nishioka, H. Kato, M. Matsumura, Collapse of anisotropic hybridization gap below $20 \mathrm{~K}$ in Kondo semiconductor $\mathrm{CeFe}_{2} \mathrm{Al}_{10}$ by pressure and magnetic field, J. Phys. Soc. Jpn. 83 (2014) 084708.

[49] H. Tanida, D. Tanaka, M. Sera, Ch. Moriyoshi, Y. Kuroiwa, T. Takesaka, T. Nishioka, H. Kato, M. Matsumura, Existence of fine structure inside spin gap in $\mathrm{CeRu}_{2} \mathrm{Al}_{10}$, J. Phys. Soc. Jpn. 79 (2010) 083701.

[50] N. Magnani, P. Santini, G. Amoretti, R. Caciuffo, Perturbative approach to $J$ mixing in $f$-electron systems: Application to actinide dioxides, Phys. Rev. B 71 (2005) 054405. 
[51] Z. Gajek, J.C. Krupa, E. Antic-Fidancev, Optical absorption spectra of the uranium (4+) ion in thorium germanate matrix, J. Phys.: Condens. Matter 9 (1997) 557.

[52] Z. Gajek, First-principles estimation of electronic structure of uranium oxychalcogenides UOY, Y $=\mathrm{S}, \mathrm{Se}, \mathrm{Te}$. Application to the INS spectra of UOS, J. Phys.: Condens. Matter 12 (2000) 415.

[53] M. Gerloch, J.H. Harding, G. Wooley, The context and application of ligand field theory, Struct. Bonding 46 (1981) 1.

[54] J. Mulak, Z. Gajek, The Effective Crystal Field Potential, Elsevier, Amsterdam, 2000.

[55] J. Otsuki, H. Kusunose, Y. Kuramoto, Theory of crystalline electric field and Kondo effect in Pr skutterudites, J. Phys. Soc. Jpn. 74 (2005) 200.

[56] The program CONDON is free software, covered by the GNU General Public License, and is available from http://www.condon.fh-aachen.de.

[57] H. Schilder, H. Lueken, Computerized magnetic studies on d, f, d-d, f-f, and d-S; f-S systems under varying ligand and magnetic fields, J. Magn. Magn. Mater. 281 (2004) 17.

[58] H. Schilder (Aachen University of Applied Sciences, Aachen, Germany), private communication.

[59] Z. Gajek, On standardization of low symmetry crystal fields, J. Phys. Chem. Solids 82 (2015) 21.

[60] G. Williams, L.L. Hirst, Crystal-field effects in solid solutions of rare earths in noble metals, Phys. Rev. 185 (1969) 407.

[61] F. Christodoulos, J.M. Dixon, A reassesment of the role played by a $5 \mathrm{~d}$ virtual bound state in the determination of crystal fields for heavy rare earth ions in gold and silver, Phys. Letters A 124 (1987) 437.

[62] Z. Gajek, M.P. Lahalle. J.C. Krupa, J. Mulak, Crystal-field effect in $\mathrm{UO}_{2}$, J. Less-Comm. Met. 139 (1988) 351.

[63] A.P. Pikul, D. Kaczorowski, Z. Gajek, J. Stępień-Damm, A. Ślebarski, M. Werwiński, A. Szajek, Giant crystal-electric-field effect and complex magnetic behavior in single-crystalline $\mathrm{CeRh}_{3} \mathrm{Si}_{2}$, Phys. Rev. B 81 (2010) 174408.

[64] R. Troć, Z. Gajek, A. Pikul, Dualism of the $5 f$ electrons of the ferromagnetic superconductor $\mathrm{UGe}_{2}$ as seen in magnetic, transport, and specific heat data, Phys. Rev. B 86 (2012) 224403.

[65] R. Troć, Z. Gajek, A. Pikul, H. Misiorek, E. Colineau, F. Wastin, Phenomenological crystal-field model of the magnetic and thermal properties of the Kondo-like system $\mathrm{UCu}_{2} \mathrm{Si}_{2}, \mathrm{Phys}$. Rev. B 88 (2013) 024416.

[66] J.A. Blanco, M. Reiffers, D. Gignoux, D. Schmitt, A.G.M. Jansen, Evidence of quadrupolar scattering in the anisotropic electrical magnetoresistivity of PrNi ${ }_{5}$, Phys. Rev. B 44 (1991) 9325.

[67] K. Kadowaki, S.B. Woods, Universal relationship of the resistivity and specific heat in heavyfermion compounds, Solid State Commun. 58 (1986) 507.

[68] Z. Kletowski, P.J. Markowski, Crystal field effects in the resistivity of the singlet ground state $\mathrm{PrIn}_{3}$ compound, Solid State Commun. 62 (1987) 299.

[69] S. Takayanagi, Crystalline electric field effects on the resistivity of $\mathrm{PrCu}_{5}$ and $\mathrm{PrCu}_{6}, \mathrm{~J}$. Phys. Soc. Jpn. 53 (1984) 676.

[70] T.A. Sayles, R.E. Baumbach, W.M. Yuhasz, M.B. Maple, Ł. Bochenek, R. Wawryk, T. Cichorek, A. Pietraszko, Z. Henkie, P.-C. Ho, Superconductivity and crystalline electric field effects in the filled skutterudite PrRu $\mathrm{As}_{12}$, Phys. Rev. B 82 (2010) 104513.

[71] A.I. Abou Aly, S. Bakanowski, N.F. Berk, J.E. Crow, T. Mihalisin, Resistive behavior in the singlet-ground-state $\mathrm{La}_{1-\mathrm{x}} \mathrm{Pr}_{\mathrm{x}} \mathrm{Sn}_{3}$, Phys. Rev. Lett. 35 (1975) 1387.

[72] Y. Kuramoto, S. Hoshino, J. Otsuki, Electronic orders induced by Kondo effect in non-Kramers f-electron systems, J. Phys. Soc. Jpn. 80 (2011) SA018.

[73] K.A. McEwen, M.J. Bull, A.M. Martin-Martin, From localised moments to non-Fermi liquids in U intermetallics, J. Phys. Soc. Jpn. 70 (2001) SA18.

[74] H.C. Walker, K.A. McEven, M.D. Le, I. Paolasini, D. Fort, X-ray resonant scattering determination of the antiferroquadrupolar ordering in $\mathrm{UPd}_{3}$ at low temperatures, J. Phys.: Condens. Matter 20 (2008) 395221.

[75] E. Gratz, A.S. Markosyan, Physical properties of $\mathrm{RCo}_{2}$ Laves phases, J. Phys.: Condens. Matter 13 (2001) R385.

[76] P. Fulde, I. Peschel, Some crystalline field effects in metals, Adv. Phys. 21 (1972) 1.

[77] R.D. Bernard, Thermoelectricity in Metals and Alloys, Taylor and Frabcis LTD, London, 1972. 
* Electronic, magnetic, thermal and transport studies for single-crystalline $\mathrm{URu}_{2} \mathrm{Al}_{10}$

* Rattling - low-frequency Einstein vibrations of the $\mathrm{U}$ atom detected in $\mathrm{URu}_{2} \mathrm{Al}_{10}$.

* Crystal field effects analyzed in the magnetic susceptibility and specific heat.

* Dual character of the $5 f$ electrons revealed in $\mathrm{URu}_{2} \mathrm{Al}_{10}$. 